\title{
Modelling and Structural Design for Parallel Umbrella-Shaped Cable-Strut Structures Based on Stationary Potential Energy Principles
}

\author{
Mingmin Ding $\mathbb{D},{ }^{1}$ Bin Luo $\mathbb{D},{ }^{2}$ Lifeng Han $\mathbb{D},{ }^{3}$ and Qianhao Shi ${ }^{4}$ \\ ${ }^{1}$ School of Civil Engineering, Nanjing Forestry University, Nanjing 210037, China \\ ${ }^{2}$ School of Civil Engineering, Southeast University, Nanjing 210096, China \\ ${ }^{3}$ Cob Development (Suzhou) Co. Ltd., Wuxi 214001, China \\ ${ }^{4}$ Elite Architectural Co. Ltd., Nanjing 210019, China
}

Correspondence should be addressed to Mingmin Ding; andyming1989seu@foxmail.com

Received 8 October 2018; Revised 26 December 2018; Accepted 10 February 2019; Published 5 May 2019

Academic Editor: Jose A. Lozano-Galant

Copyright (C) 2019 Mingmin Ding et al. This is an open access article distributed under the Creative Commons Attribution License, which permits unrestricted use, distribution, and reproduction in any medium, provided the original work is properly cited.

\begin{abstract}
A method for the modelling and structural design of a parallel umbrella-shaped cable-strut structure (PUSC) is presented. First, simplified calculation models of a PUSC are built. Next, based on the principle of stationary potential energy, the relationships among the cable sectional areas, prestress forces, vector height, sag height, overall displacement, and local deformation are proposed. Then, the static responses of the PUSC under vertical loads and wind loads are put forward. Finally, a calculation model of a $100 \mathrm{~m}$-span PUSC is developed and optimized to verify the feasibility of the proposed method. The results show that when the combinations of the loading, variation ranges of the vector height and sag height, and material properties of the components are given, the sectional areas of the cables, dimensions of the inner strut, and prestress forces of these components can be obtained. A greater external load requires a corresponding increase in vector height and sag height to increase the overall stiffness, leading to larger sectional dimensions of the components and a greater prestress of the entire structure. Therefore, the total weight of the cables and inner struts are determined. Moreover, because the weight of the cables decreases and the weight of the inner struts increases as the vector height and sag height increase, the total weight of the cables and struts decreases sharply during the initial stage, decreases gradually during the second stage, and increases slowly during the last stage after reaching the minimum value. For the optimal design of the calculation model, using the vector height and sag height as design variables provides an adequate geometric stiffness and a suitable prestress for the PUSC to fulfill the requirements of all the loading combinations.
\end{abstract}

\section{Introduction}

In the last two decades, cable-strut structures, such as cable-dome structures and cable-truss structures, have been widely used in various large-span projects [1] and aviation antennas [2]. A cable-strut structure is a type of prestressed structure that includes a large number of flexible cables and struts. The major advantages of cable-strut structures are as follows: (1) large structural rigidity with the introduction of prestress forces in cables and struts, (2) low weight of the entire structure with high-strength metal materials used as the cables, (3) slender struts with compressive forces added in the axial direction, and (4) simple node configurations with adjacent components connected by hinged joints. As society develops, more complex cablestrut structures will be used to cover wider spans with relatively lower project costs. For these applications, the parallel umbrella-shaped cable-strut structure (PUSC) is a well-suited approach. A PUSC is a double-layer cable-strut roof system, and each substructure of a PUSC is a triangular cable net with one central compressive strut. Due to their long spans, high clearances, low construction costs, and elegant shapes, PUSCs have been widely used in various large-span projects, such as the AstroMesh antennas [3] and the roof of the Sony Centre in Berlin's Potsdamer Square [4]. 
Unlike traditional steel structures [5], the axial pretension forces in the cables and axial precompression forces in the struts provide PUSCs the rigidity and ability to resist external loads [6]. Therefore, the structural design of PUSCs is related to both the structural shape and the initial prestress distribution and requires in-depth study.

Currently, most research on structural design is focused on rigid structures, and many theories and methods have been proposed based on energy principles, e.g., a combination of the total complementary energy minimization theory and a modified sequential quadratic programming (SQP) algorithm discussed by Ohkubo et al. [7], a metaheuristic algorithm developed by Toklu et al. [8], and a particle swarm optimization (PSO) algorithm presented by Temür et al. [9]. However, research on the structural design of large-span steel structures is lacking, with most of the current studies predominantly limited to trusses $[10,11]$, as discussed by Kawamura et al. [12].

Numerous studies have been conducted on the formfinding and force-finding of cable-strut structures, such as the force-density method presented by Schek [13], the dynamic relaxation method proposed by Motro et al. [14], the nonlinear force method (NFM) presented by Luo [15], and the nonlinear dynamic finite element method (NDFEM) proposed by Luo et al. [1]. More recently, Zhou et al. [16] proposed the modified double singular value decomposition method to acquire feasible prestress states of cable-strut structures without changing its predefined shape.

Most of these works involve form-finding and forcefinding and are barely related to the structural design of PUSCs. These studies are mainly based on given prestress distributions or given configuration modes of entire structures with predetermined support node locations. If all of these parameters are not given, the structural design will be more intricate, yet such a situation is likely, especially in the initial design stage. However, few studies have focused on design problems under these conditions, and these studies have predominantly focused on antennas [17].

Considering these aspects, this paper addresses a structural design method that can be applied to PUSCs without determining the initial prestress distributions and even without knowing the sectional areas of the cables. The simplified models and basic assumptions are presented first, followed by the calculation models in different states. Subsequently, the relations among the forces of the cables and struts, the sectional areas of the cables, the specifications of the struts, the vector height, and the sag height are derived. Using the minimum total weight of all the cables and struts as the design objective and the values of vector height and sag height as the design variables, the structural design, including the initial structural shape, the initial prestress, and the parameters of the structural components, of a PUSC can be obtained. Finally, the roof of the Shijiazhuang International Exhibition Centre is used as an example to verify the applicability of the structural design method. This method is capable of producing a PUSC with a minimal total weight and reasonable mechanical performance.

\section{Description of the PUSC}

2.1. Simplified Models and Basic Assumptions. A PUSC is a cable-strut structure that comprises $n$ substructures (where $n$ is a positive integer), and each substructure consists of two ridge cables, two diagonal cables, and one inner strut. The joints between adjacent components are hinged. The simplified calculation model of a PUSC is shown in Figure 1(a), and the simplified model of a substructure is shown in Figure 1(b).

2.2. Calculation Models in Different States. According to the loads added on the structure, a PUSC has three predominant states: the initial geometric state, the initial prestress state, and the loading equilibrium state.

(1) The initial geometric state is an equilibrium state without building prestress or adding external loads. This state corresponds to the natural form of a PUSC under its selfweight and is primarily controlled by its initial geometric shape.

(2) The initial prestress state is an equilibrium state that considers the effect of prestress only. The initial prestress state of a PUSC is the basis for deriving the structural forces and deformations under complex external loads and is primarily controlled by the initial geometric shape and the distribution mode of the prestress. The simplified calculation model for the initial prestress state of a PUSC is shown in Figure 2.

(3) The loading equilibrium state is an equilibrium state that considers the effect of both the prestress and external loads. This state is primarily controlled by the initial geometric shape, the prestress, and the external loads. The external structural loads include vertical loads (e.g., dead load, live load, and snow load) and wind loads (e.g., suction wind load and pressure wind load), where the vertical loads are directed towards the ground, the suction wind load is directed upward and perpendicular to the ridge cables, and the pressure wind load is directed downward and perpendicular to the ridge cables. In this case, to determine the structural response of a PUSC in the loading equilibrium state, the mechanical theories of PUSCs under vertical loads and wind loads must be determined first.

Because the substructures of a PUSC are mutually parallel, a load (i.e., a vertical load or a wind load) added to the ridge cables of the PUSC is uniformly distributed. Details of the deformations of a PUSC under different loads are shown in Figure 3.

\section{Structural Performance of PUSCs Based on the Stationary Potential Energy Principle}

3.1. Stationary Potential Energy Principle. Different displacements may occur under different loading conditions for a cable-strut structure. The total potential energy of the structure can be expressed as

$$
\Pi=U+P
$$

For the linear elastic cable elements, only axial deformation is considered. In this case, $U$ is expressed as 


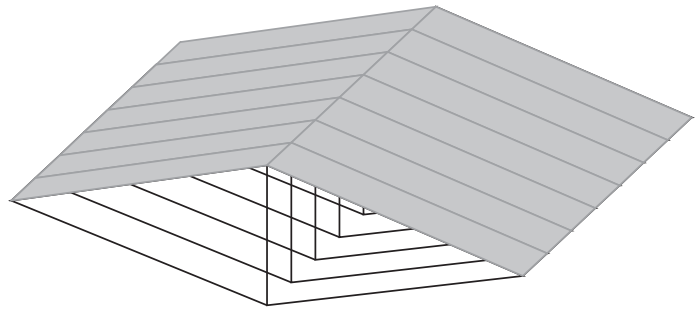

(a)

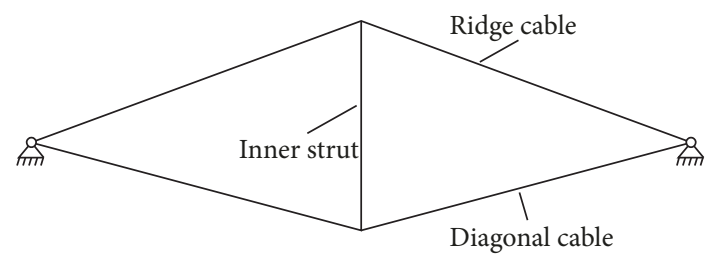

(b)

FIGURE 1: Simplified models of a PUSC. (a) The entire model. (b) Calculation model of a substructure.

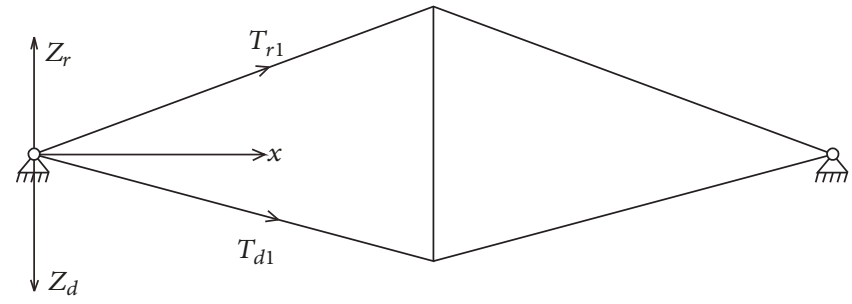

FIGURE 2: Simplified calculation model for the initial prestress state of a PUSC.

$$
\begin{aligned}
U & =\left(T_{r 1}+\frac{1}{2} \Delta T_{r}\right) \Delta l_{r}+\left(T_{d 1}+\frac{1}{2} \Delta T_{d}\right) \Delta l_{d} \\
\Delta T_{r} & =\frac{E A_{r} \Delta l_{r}}{l_{r}} \\
\Delta T_{d} & =\frac{E A_{d} \Delta l_{d}}{l_{d}}
\end{aligned}
$$

When adding an external load, the potential energy of the entire structure is

$$
P=-\sum R \Delta X
$$

Considering (2), (3), (4), and (5), the expression of the total potential energy of the structure is

$$
\begin{aligned}
\Pi= & \left(\frac{H_{r 1} \sqrt{d_{1}^{2}+L^{2}}}{L}+\frac{E A_{r} \Delta l_{r}}{2 l_{r}}\right) \Delta l_{r} \\
& +\left(\frac{H_{r 1} d_{1} \sqrt{d_{2}^{2}+L^{2}}}{L d_{2}}+\frac{E A_{d} \Delta l_{d}}{2 l_{d}}\right) \Delta l_{d}-\Sigma R \Delta X
\end{aligned}
$$

Applying the principle of stationary potential energy [18], (7) can be obtained to describe the static equilibrium equation of the structure.

$$
\frac{\partial \Pi}{\partial w}=0
$$
State. According to Figure 2, the configuration equations for the ridge cables and the diagonal cables in the initial prestress state are defined as follows:

$$
\begin{aligned}
& Z_{r}=\frac{d_{1}}{L} x \\
& Z_{d}=\frac{d_{2}}{L} x
\end{aligned}
$$

Assuming that $H_{r 1}$ is known, $T_{r 1}$ and $T_{d 1}$ are

$$
\begin{aligned}
& T_{r 1}=\frac{H_{r 1} \sqrt{d_{1}^{2}+L^{2}}}{L} \\
& T_{d 1}=\frac{H_{r 1} d_{1} \sqrt{d_{2}^{2}+L^{2}}}{L d_{2}}
\end{aligned}
$$

Hence, $\Pi$ is

$$
\begin{aligned}
\Pi= & \left(\frac{H_{r 1} \sqrt{d_{1}^{2}+L^{2}}}{L}+\frac{1}{2} \frac{E A_{r} \Delta l_{r}}{l_{r}}\right) \Delta l_{r} \\
& +\left(\frac{H_{r 1} d_{1} \sqrt{d_{2}^{2}+L^{2}}}{L d_{2}}+\frac{1}{2} \frac{E A_{d} \Delta l_{d}}{l_{d}}\right) \Delta l_{d} \\
& -\sum R \Delta X
\end{aligned}
$$

and $H_{d 1}$ is

$$
H_{d 1}=\frac{H_{r 1} d_{1}}{d_{2}}
$$

3.3. Structural Performance of PUSCs in the Vertical Loading Condition. Under a vertical load, the ridge cables of parallel 


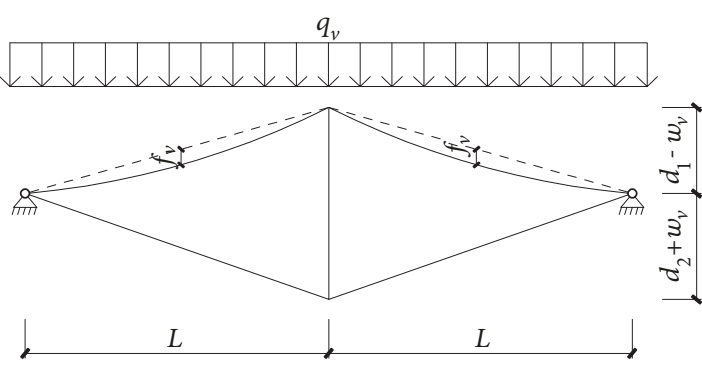

(a)

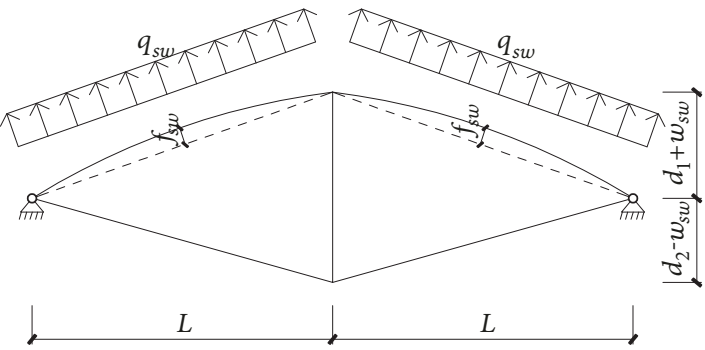

(b)

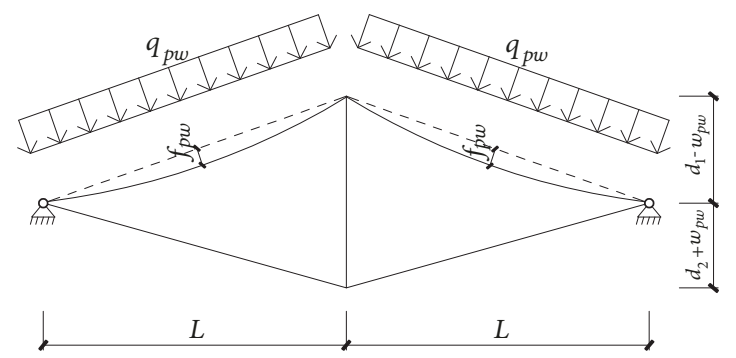

(c)

FIGURE 3: Deformations of a PUSC under different loads. (a) Uniformly distributed vertical load. (b) Uniformly distributed suction wind load. (c) Uniformly distributed pressure wind load.

PUSCs are directed vertically downwards. When adding the uniform vertical line load, $q_{v}$, the deformations of the ridge cables are caused by $w_{v}$ and $f_{v}$, and the deformations of the diagonal cables are caused by $w_{v}$ only. In this case, $T_{r 2, v}$ is

$$
T_{r 2, v}=T_{r 1, v}+E A_{r, v} \frac{\Delta l_{r, v}}{\sqrt{L^{2}+d_{1}^{2}}}
$$

Because a cable follows a parabolic shape under a uniform line load [19], $f_{v}$ can be expressed as

$$
f_{v}=\frac{q_{v} L^{2}}{8 H_{r 2, v}}
$$

The deformation of the ridge cables is

$$
u_{r, v}=u_{r 1, v}+u_{r 2, v}
$$

The deformations caused by $w_{v}$ and $f_{v}$ can be expressed as follows:

$$
\begin{aligned}
& u_{r 1, v}=-\frac{w_{v}}{L} x \\
& u_{r 2, v}=-\frac{4 f_{v} x(L-x)}{L^{2}}
\end{aligned}
$$

where the elongation of the ridge cables caused by $w_{v}$ is

$$
\begin{aligned}
& \Delta l_{r 1, v} \\
& \quad=\int_{0}^{L}\left(\sqrt{1+\left(Z_{r}^{\prime}+u_{r 2, v}{ }^{\prime}\right)^{2}}-\sqrt{\left(1+\left(Z_{r}^{\prime}\right)^{2}\right.}\right) d x \\
& \quad=\sqrt{L^{2}+\left(d_{1}-w_{v}\right)^{2}}-\sqrt{L^{2}+d_{1}^{2}}
\end{aligned}
$$

The elongation of the ridge cables caused by $f_{v}$ is

$$
\begin{aligned}
& \Delta l_{r 2, v} \\
& \quad=\int_{0}^{L}\left(\sqrt{1+\left(Z_{r}^{\prime}+u_{r 2, v}{ }^{\prime}\right)^{2}}-\sqrt{\left(1+\left(Z_{r}^{\prime}\right)^{2}\right.}\right) \mathrm{d} x \\
& \quad \approx \frac{8 f_{v}^{2}}{3 L}
\end{aligned}
$$

and the total elongation of a ridge cable is

$$
\begin{aligned}
\Delta l_{r, v} & =\Delta l_{r 1, v}+\Delta l_{r 2, v} \\
& =\sqrt{L^{2}+\left(d_{1}-w_{v}\right)^{2}}-\sqrt{L^{2}+d_{1}^{2}}+\frac{8 f_{v}^{2}}{3 L}
\end{aligned}
$$

The deformation of the diagonal cables can be expressed as

$$
u_{d, v}=\frac{w_{v}}{L} x
$$

The elongation of the diagonal cables is caused by $f_{v}$ only and can be expressed as

$$
\begin{aligned}
& \Delta l_{d, v} \\
& \quad=\int_{0}^{L}\left(\sqrt{1+\left(Z_{d}^{\prime}+u_{d, v}{ }^{2}\right)^{2}}-\sqrt{\left(1+\left(Z_{d}^{\prime}\right)^{2}\right.}\right) \mathrm{d} x \\
& \quad=\sqrt{L^{2}+\left(d_{2}+w_{v}\right)^{2}}-\sqrt{L^{2}+d_{2}^{2}}
\end{aligned}
$$


Then, the potential energy of the total structure can be expressed as

$$
\begin{aligned}
\int_{0}^{L} q_{v}\left(-\frac{4 f_{v} x(L-x)}{L^{2}}-\frac{w_{v}}{L} x\right) \mathrm{d} x \\
=-\frac{2 q_{v} L f_{v}}{3}-\frac{q_{v} L w_{v}}{2}
\end{aligned}
$$

Combining (6), (7), (21), and (24), the total energy of the entire structure is

$$
\begin{aligned}
& \Pi=\left\{\frac{8 f_{v}{ }^{2}}{3 L}+L\left[\sqrt{1+\left(-\frac{w_{v}}{L}+\frac{d_{1}}{L}\right)^{2}}-\sqrt{1+\frac{d_{1}^{2}}{L^{2}}}\right]\right\} \\
& \left\{\frac{H_{r 1, v} \sqrt{L^{2}+d_{1}^{2}}}{L}+E A_{r, v}\right. \\
& \left.\frac{8 f_{v}^{2} / 3 L+L\left[\sqrt{1+\left(-w_{v} / L+d_{1} / L\right)^{2}}-\sqrt{1+d_{1}^{2} / L^{2}}\right]}{2 \sqrt{L^{2}+d_{1}^{2}}}\right\} \\
& +\left\{\frac{H_{r 1, v} d_{1} \sqrt{L^{2}+d_{2}^{2}}}{d_{2} L}+E A_{d, v}\right. \\
& \left.\frac{L\left[\sqrt{1+\left(w_{v} / L+d_{2} / L\right)^{2}}-\sqrt{1+d_{2}^{2} / L^{2}}\right]}{2 \sqrt{L^{2}+d_{2}^{2}}}\right\} \\
& \times L\left[\sqrt{1+\left(\frac{w_{v}}{L}+\frac{d_{2}}{L}\right)^{2}}-\sqrt{1+\frac{d_{2}^{2}}{L^{2}}}\right]-\frac{2 q_{v} L f_{v}}{3} \\
& -\frac{q_{v} L w_{v}}{2}
\end{aligned}
$$

According to (7), the partial derivative of (25) is

$$
\begin{aligned}
-\frac{L q_{v}}{2} & -\frac{E A_{r, v} d_{1}}{\sqrt{L^{2}+d_{1}^{2}}} \\
& -\frac{8 E A_{r, v} f_{v}^{2} d_{1}}{3 L^{2} \sqrt{L^{2}+d_{1}^{2}} \sqrt{1+\left(-w_{v} / L+d_{1} / L\right)^{2}}} \\
& -\frac{H_{r 1, v} d_{1} \sqrt{L^{2}+d_{1}^{2}}}{L^{2} \sqrt{1+\left(-w_{v} / L+d_{1} / L\right)^{2}}} \\
+ & \frac{E A_{r, v} d_{1}}{L \sqrt{1+\left(-w_{v} / L+d_{1} / L\right)^{2}}}+\frac{E A_{d, v} d_{2}}{\sqrt{L^{2}+d_{2}^{2}}}
\end{aligned}
$$

$$
\begin{aligned}
& +\frac{H_{r 1, v} d_{1} \sqrt{L^{2}+d_{2}^{2}}}{L^{2} \sqrt{1+\left(w_{v} / L+d_{2} / L\right)^{2}}} \\
& -\frac{E A_{d, v} d_{2}}{L \sqrt{1+\left(w_{v} / L+d_{2} / L\right)^{2}}}+\frac{E A_{r, v} w_{v}}{\sqrt{L^{2}+d_{1}^{2}}} \\
& -\frac{E A_{r, v} w_{v}}{L \sqrt{1+\left(-w_{v} / L+d_{1} / L\right)^{2}}} \\
& +\frac{8 E A_{r, v} f_{v}^{2} w_{v}}{3 L^{2} \sqrt{L^{2}+d_{1}^{2}} \sqrt{1+\left(-w_{v} / L+d_{1} / L\right)^{2}}} \\
& +\frac{E A_{d, v} w_{v}}{\sqrt{L^{2}+d_{2}^{2}}-\frac{E A_{d, v} w_{v}}{L \sqrt{1+\left(w_{v} / L+d_{2} / L\right)^{2}}}} \\
& +\frac{H_{r 1, v} d_{1} w_{v} \sqrt{L^{2}+d_{2}^{2}}}{L^{2} d_{2} \sqrt{1+\left(w_{v} / L+d_{2} / L\right)^{2}}} \\
& +L_{r 1, v}^{2} \sqrt{1+\left(-w_{v} / L+d_{1} / L\right)^{2}}
\end{aligned}
$$

Assuming that $q_{v}=0, f_{v}=0$, and $w_{v}=0$, the left side of the equal sign in (26) can be expressed as

$$
\begin{gathered}
-\frac{E A_{r, v} d_{1}}{\sqrt{L^{2}+d_{1}^{2}}}-\frac{H_{r 1, v} d_{1} \sqrt{L^{2}+d_{1}^{2}}}{L \sqrt{L^{2}+d_{1}^{2}}}+\frac{E A_{r, v} d_{1}}{\sqrt{L^{2}+d_{1}^{2}}} \\
+\frac{E A_{d, v} d_{2}}{\sqrt{L^{2}+d_{2}^{2}}}+\frac{H_{r 1, v} d_{1} \sqrt{L^{2}+d_{2}^{2}}}{L \sqrt{L^{2}+d_{2}^{2}}}-\frac{E A_{d, v} d_{2}}{\sqrt{L^{2}+d_{2}^{2}}}
\end{gathered}
$$

After performing these computations, (27) is equal to 0 . In this case, (26) is correct, i.e., (26) meets the principle of stationary potential energy.

If $w_{v}$ is small relative to $L$ and assuming that $w_{v} / L \approx 0,(26)$ is

$$
\begin{gathered}
-\frac{L q_{v}}{2}-\frac{8 E A_{r, v} f_{v}^{2} d_{1}}{3 L\left(L^{2}+d_{1}^{2}\right)}+\frac{8 E A_{r, v} f_{v}^{2} w_{v}}{3 L\left(L^{2}+d_{1}^{2}\right)}+\frac{E A_{r, v} d_{1}^{2} w_{v}}{\left(L^{2}+d_{1}^{2}\right)^{3 / 2}} \\
+\frac{E A_{d, v} d_{2}^{2} w_{v}}{\left(L^{2}+d_{2}^{2}\right)^{3 / 2}}+\frac{H_{r 1, v} d_{1} w_{v}}{L d_{2}}+\frac{H_{r 1, v} w_{v}}{L}=0
\end{gathered}
$$

By substituting (15) into (28), $w_{v}$ is

$$
w_{v}=\frac{L q_{v} / 2+E A_{r} q_{v}^{2} L^{3} d_{1} / 24 H_{r 2, v}^{2}\left(L^{2}+d_{1}^{2}\right)}{E A_{r} q_{v}^{2} L^{3} / 24 H_{r 2, v}^{2}\left(L^{2}+d_{1}^{2}\right)+E A_{r} d_{1}^{2} /\left(L^{2}+d_{1}^{2}\right)^{3 / 2}+E A_{d} d_{2}^{2} /\left(L^{2}+d_{2}^{2}\right)^{3 / 2}+H_{r 1, v}\left(d_{1}+d_{2}\right) / L d_{2}}
$$


Assuming that all of the cables reach their design strength after loading and that the values of $f_{v}$ and $w_{v}$ are both given, the following equations can be obtained:

$$
\begin{aligned}
& T_{d 2, v}=A_{d, v} \sigma_{c o n}=T_{d 1, v}+E A_{d, v} \frac{\Delta L_{d, v}}{L_{d, v}} \\
& =\frac{H_{r 1, v} d_{1} \sqrt{d_{2}^{2}+L^{2}}}{L d_{2}}+\frac{E A_{d, v} \sqrt{\left(d_{2}+w_{v}\right)^{2}+L^{2}}}{\sqrt{d_{2}^{2}+L^{2}}} \\
& -E A_{d, v} \\
& H_{r 1, v}=\frac{A_{r, v}\left[\left(\sigma_{c o n}+E\right) L \sqrt{L^{2}+d_{1}^{2}}-E L \sqrt{L^{2}+\left(d_{1}-w_{v}\right)^{2}}-(8 / 3) E f_{v}^{2}\right]}{\left(L^{2}+d_{1}^{2}\right)} \\
& A_{d, v}=\frac{A_{r, v} d_{1}\left(d_{2}^{2}+L^{2}\right)\left[\sigma_{c o n} \sqrt{L^{2}+d_{1}^{2}}-E\left(\sqrt{L^{2}+\left(d_{1}-w_{v}\right)^{2}}-\sqrt{L^{2}+d_{1}^{2}}+8 f_{v}^{2} / 3 L\right)\right]}{d_{2}\left(L^{2}+d_{1}^{2}\right)\left[\left(\sigma_{c o n}+E\right) \sqrt{d_{2}^{2}+L^{2}}-E \sqrt{\left(d_{2}+w_{v}\right)^{2}+L^{2}}\right]}
\end{aligned}
$$

$$
\begin{aligned}
H_{r 1, v} & =\sigma_{c o n} A_{r, v} \frac{L}{\sqrt{L^{2}+d_{1}^{2}}}-E A_{r, v} \frac{L \Delta l_{r, v}}{L^{2}+d_{1}^{2}} \\
& =A_{r, v}\left(\sigma_{c o n} \frac{L}{\sqrt{L^{2}+d_{1}^{2}}}-\frac{E L \Delta l_{r, v}}{L^{2}+d_{1}^{2}}\right)
\end{aligned}
$$

In this case, the sectional areas of the ridge cables and diagonal cables in the vertical loading condition, $A_{r, v}$ and $A_{d, v}$, can be denoted by using (15), (29), and (33).

$$
A_{r, v}=\frac{L q_{v}}{2 X^{*}}
$$

$$
A_{d, v}=\frac{L q_{v} d_{1}\left(d_{2}^{2}+L^{2}\right)\left[\sigma_{c o n} \sqrt{L^{2}+d_{1}^{2}}-E\left(\sqrt{L^{2}+\left(d_{1}-w_{v}\right)^{2}}-\sqrt{L^{2}+d_{1}^{2}}+8 f_{v}^{2} / 3 L\right)\right]}{2 X^{*} d_{2}\left(L^{2}+d_{1}^{2}\right)\left[\left(\sigma_{c o n}+E\right) \sqrt{d_{2}^{2}+L^{2}}-E \sqrt{\left(d_{2}+w_{v}\right)^{2}+L^{2}}\right]}
$$

where

$$
\begin{gathered}
X^{*}=\frac{8 E f_{v}^{2}\left(w_{v}-d_{1}\right)}{3 L\left(L^{2}+d_{1}^{2}\right)}+\frac{w_{v}\left(d_{1}+d_{2}\right)\left[\left(\sigma_{c o n}+E\right) \sqrt{L^{2}+d_{1}^{2}}-E \sqrt{L^{2}+\left(d_{1}-w_{v}\right)^{2}}-8 E f_{v}^{2} / 3 L\right]}{d_{2}\left(L^{2}+d_{1}^{2}\right)} \\
+\frac{E w_{v} d_{1} d_{2}\left[\left(\sigma_{c o n}+E\right) \sqrt{L^{2}+d_{1}^{2}}-E \sqrt{L^{2}+\left(d_{1}-w_{v}\right)^{2}}-8 E f_{v}^{2} / 3 L\right]}{\left(L^{2}+d_{1}^{2}\right)\left[\left(\sigma_{c o n}+E\right)\left(L^{2}+d_{2}^{2}\right)-E \sqrt{L^{2}+d_{2}^{2}} \sqrt{\left(w_{v}+d_{2}\right)^{2}+L^{2}}\right]}+\frac{E w_{v} d_{1}^{2}}{\left(L^{2}+d_{1}^{2}\right)^{1.5}}
\end{gathered}
$$

3.4. Structural Performance of PUSCs in the Suction Wind Loading Condition. In contrast to the vertical loads, the ridge cables move upwards perpendicular to their lengths under suction wind loads. The maximum local deformation of the ridge cables is

$$
f_{s w}=\frac{q_{s w}\left(L^{2}+d_{1}^{2}\right)}{8 T_{r 2, s w}}=\frac{q_{s w} L \sqrt{L^{2}+d_{1}^{2}}}{8 H_{r 2, s w}}
$$


The elongation of the ridge cables is

$$
\begin{aligned}
\Delta l_{r, s w}= & \Delta l_{r 1, s w}+\Delta l_{r 2, s w} \\
= & \sqrt{L^{2}+\left(d_{1}-w_{s w}\right)^{2}}-\sqrt{L^{2}+d_{1}^{2}} \\
& +\frac{8 f_{s w}^{2}}{3 \sqrt{L^{2}+d_{1}^{2}}}
\end{aligned}
$$

Hence, the potential energy of the total structure is

$$
\begin{aligned}
& \int_{0}^{L / \cos (\theta)} q_{s w}\left(-\frac{4 f_{s w} x(L / \cos (\theta)-x)}{(L / \cos (\theta))^{2}}\right. \\
& \left.-\frac{w_{s w}(\cos (\theta))^{2} x}{L}\right) \mathrm{d} x \\
& =-\frac{2 f_{s w} q_{s w} \sqrt{L^{2}+d_{1}^{2}}}{3}-\frac{L q_{s w} w_{s w}}{2}
\end{aligned}
$$

Combining (6), (7), (38), and (39), $w_{s w}$ is

$$
w_{s w}=\frac{L q_{s w} / 2+E A_{r, s w} q_{s w}^{2} L^{2} d_{1} / 24 H_{r 2, s w}^{2} \sqrt{\left(L^{2}+d_{1}^{2}\right)}}{E A_{r, s w} q_{s w}^{2} L^{2} / 24 H_{r 2, s w}^{2} \sqrt{\left(L^{2}+d_{1}^{2}\right)}+E A_{r, s w} d_{1}^{2} /\left(L^{2}+d_{1}^{2}\right)^{3 / 2}+E A_{d, s w} d_{2}^{2} /\left(L^{2}+d_{2}^{2}\right)^{3 / 2}+H_{r 1, s w}\left(d_{1}+d_{2}\right) / L d_{2}}
$$

Assuming that all of the cables reach their design strength after loading and that the values of $f_{s w}$ and $w_{s w}$ are both given, the following equations can be obtained:

$$
\begin{aligned}
& T_{d 2, s w}=A_{d, s w} \sigma_{c o n}=T_{d 1, s w}+E A_{d, s w} \frac{\Delta L_{d, s w}}{L_{d, s w}}=\frac{H_{r 1, s w} d_{1} \sqrt{d_{2}^{2}+L^{2}}}{L d_{2}}+\frac{E A_{d, s w} \sqrt{\left(d_{2}+w_{s w}\right)^{2}+L^{2}}}{\sqrt{d_{2}^{2}+L^{2}}}-E A_{d, s w} \\
& H_{r 1, s w}=\sigma_{c o n} A_{r, s w} \frac{L}{\sqrt{L^{2}+d_{1}^{2}}}-E A_{r, s w} \frac{L \Delta l_{r, s w}}{L^{2}+d_{1}^{2}}=A_{r, s w}\left(\sigma_{c o n} \frac{L}{\sqrt{L^{2}+d_{1}^{2}}}-\frac{E L \Delta l_{r, s w}}{L^{2}+d_{1}^{2}}\right) \\
& =A_{r, s w}\left(\frac{\left(\sigma_{c o n}+E\right) L}{\sqrt{L^{2}+d_{1}^{2}}}-\frac{8 E L f_{s w}^{2}}{3\left(L^{2}+d_{1}^{2}\right)^{1.5}}-\frac{E L \sqrt{L^{2}+\left(d_{1}-w_{s w}\right)^{2}}}{L^{2}+d_{1}^{2}} \mid\right. \text { height21.65997ptdepth16.56ptwidth0pt) } \\
& A_{d, s w}=A_{r, s w} \frac{d_{1}\left(d_{2}^{2}+L^{2}\right)\left[\left(\sigma_{c o n}+E\right) \sqrt{L^{2}+d_{1}^{2}}-E \sqrt{L^{2}+\left(d_{1}-w_{s w}\right)^{2}}-8 E f_{s w}^{2} / 3 \sqrt{L^{2}+d_{1}^{2}}\right]}{d_{2}\left(L^{2}+d_{1}^{2}\right)\left[\left(\sigma_{c o n}+E\right) \sqrt{d_{2}^{2}+L^{2}}-E \sqrt{\left(d_{2}+w_{s w}\right)^{2}+L^{2}}\right]}
\end{aligned}
$$

In this case, the sectional areas of the ridge cables and diagonal cables in the suction wind loading condition can be denoted by using (37), (40), and (43).

$$
\begin{aligned}
& A_{r, s w}=\frac{L q_{s w}}{2 Y^{*}} \\
& A_{d, s w}=\frac{L q_{s w} d_{1}\left(d_{2}^{2}+L^{2}\right)\left[\sigma_{c o n} \sqrt{L^{2}+d_{1}^{2}}-E\left(\sqrt{L^{2}+\left(d_{1}-w_{s w}\right)^{2}}-\sqrt{L^{2}+d_{1}^{2}}+8 f_{s w}^{2} / 3 \sqrt{L^{2}+d_{1}^{2}}\right)\right]}{2 Y^{*} d_{2}\left(L^{2}+d_{1}^{2}\right)\left[\left(\sigma_{c o n}+E\right) \sqrt{d_{2}^{2}+L^{2}}-E \sqrt{\left(d_{2}+w_{s w}\right)^{2}+L^{2}}\right]}
\end{aligned}
$$

where 


$$
\begin{aligned}
Y^{*}= & \frac{E\left(3 w_{s w} d_{1}^{2} d_{2}-8 f_{s w}^{2} w_{s w} d_{1}-8 f_{s w}^{2} d_{1} d_{2}\right)}{3 d_{2}\left(L^{2}+d_{1}^{2}\right)^{3 / 2}}+\frac{\left(d_{1}+d_{2}\right)\left[\left(\sigma_{c o n}+E\right) w_{s w} \sqrt{L^{2}+d_{1}^{2}}-E w_{s w} \sqrt{L^{2}+\left(d_{1}-w_{s w}\right)^{2}}\right]}{d_{2}\left(L^{2}+d_{1}^{2}\right)} \\
& +\frac{E d_{1} d_{2} w_{s w}\left[\left(\sigma_{c o n}+E\right) \sqrt{L^{2}+d_{1}^{2}}-E \sqrt{L^{2}+\left(d_{1}-w_{s w}\right)^{2}}-8 E f_{s w}^{2} / 3 \sqrt{L^{2}+d_{1}^{2}}\right]}{\left(L^{2}+d_{1}^{2}\right) \sqrt{L^{2}+d_{2}^{2}}\left[\left(\sigma_{c o n}+E\right) \sqrt{d_{2}^{2}+L^{2}}-E \sqrt{\left(d_{2}+w_{s w}\right)^{2}+L^{2}}\right]}
\end{aligned}
$$

3.5. Structural Performance of PUSCs in the Pressure Wind Loading Condition. Under a pressure wind load, the ridge cables move downwards perpendicular to their lengths with a maximum local deformation as follows:

$$
f_{p w}=\frac{q_{p w}\left(L^{2}+d_{1}^{2}\right)}{8 T_{r 2, p w}}=\frac{q_{p w} L \sqrt{L^{2}+d_{1}^{2}}}{8 H_{r 2, p w}}
$$

The elongation of the ridge cables is

$$
\Delta l_{r, p w}=\Delta l_{r 1, p w}+\Delta l_{r 2, p w}
$$

$$
\begin{aligned}
= & \frac{8 f_{p w}^{2}}{3 \sqrt{L^{2}+d_{1}^{2}}} \\
& +L\left(-\sqrt{1+\frac{d_{1}^{2}}{L^{2}}}+\sqrt{1+\left(-\frac{w_{p w}}{L}+\frac{d_{1}}{L}\right)^{2}}\right)
\end{aligned}
$$

Afterwards, the vertical displacement of the inner strut can be expressed using the same derivation process as that used for the suction wind loading condition.

$$
w_{p w}=\frac{L q_{p w} / 2+E A_{r, p w} q_{p w}^{2} L^{2} d_{1} / 24 H_{r 2, p w}^{2} \sqrt{\left(L^{2}+d_{1}^{2}\right)}}{E A_{r, p w} q_{p w}^{2} L^{2} / 24 H_{r 2, p w}^{2} \sqrt{\left(L^{2}+d_{1}^{2}\right)}+E A_{r, p w} d_{1}^{2} /\left(L^{2}+d_{1}^{2}\right)^{3 / 2}+E A_{d, p w} d_{2}^{2} /\left(L^{2}+d_{2}^{2}\right)^{3 / 2}+H_{r 1, p w}\left(d_{1}+d_{2}\right) / L d_{2}}
$$

Assuming that all of the cables reach their design strength after loading, the following equations are obtained:

$$
\begin{aligned}
& T_{d 2, p w}=A_{d, p w} \sigma_{c o n}=T_{d 1, p w}+E A_{d, p w} \frac{\Delta L_{d, p w}}{L_{d, p w}}=\frac{H_{r 1, p w} d_{1} \sqrt{d_{2}^{2}+L^{2}}}{L d_{2}}+\frac{E A_{d, p w} \sqrt{\left(d_{2}+w_{p w}\right)^{2}+L^{2}}}{\sqrt{d_{2}^{2}+L^{2}}}-E A_{d, p w} \\
& H_{r 1, p w}=\sigma_{c o n} A_{r, p w} \frac{L}{\sqrt{L^{2}+d_{1}^{2}}}-E A_{r, p w} \frac{L \Delta l_{r, p w}}{L^{2}+d_{1}^{2}}=A_{r, p w}\left(\sigma_{c o n} \frac{L}{\sqrt{L^{2}+d_{1}^{2}}}-\frac{E L \Delta l_{r, p w}}{L^{2}+d_{1}^{2}}\right) \\
& =A_{r, p w}\left(\frac{\left(\sigma_{c o n}-E\right) L}{\sqrt{L^{2}+d_{1}^{2}}}-\frac{8 E L f_{p w}^{2}}{3\left(L^{2}+d_{1}^{2}\right)^{1.5}}+\frac{E L \sqrt{L^{2}+\left(d_{1}-w_{p w}\right)^{2}}}{L^{2}+d_{1}^{2}}\right) \\
& A_{d, p w}=A_{r, p w} \frac{d_{1}\left(d_{2}^{2}+L^{2}\right)\left[\sigma_{c o n} \sqrt{L^{2}+d_{1}^{2}}-E \sqrt{L^{2}+\left(d_{1}-w_{p w}\right)^{2}}+E \sqrt{L^{2}+d_{1}^{2}}-8 E f_{p w}^{2} / 3 \sqrt{L^{2}+d_{1}^{2}}\right]}{d_{2}\left(L^{2}+d_{1}^{2}\right)\left[\left(\sigma_{c o n}+E\right) \sqrt{d_{2}^{2}+L^{2}}-E \sqrt{\left(d_{2}+w_{p w}\right)^{2}+L^{2}}\right]}
\end{aligned}
$$


In this case, the sectional areas of ridge cables and diagonal cables in the pressure wind loading condition can be denoted by using (47), (49), and (52).

$$
A_{r, p w}=\frac{L q_{p w}}{2 Z^{*}}
$$

$$
A_{d, p w}=\frac{L q_{p w} d_{1}\left(d_{2}^{2}+L^{2}\right)\left[\sigma_{c o n} \sqrt{L^{2}+d_{1}^{2}}-E\left(\sqrt{L^{2}+\left(d_{1}-w_{p w}\right)^{2}}-\sqrt{L^{2}+d_{1}^{2}}+8 f_{p w}^{2} / 3 \sqrt{L^{2}+d_{1}^{2}}\right)\right]}{2 Z^{*} d_{2}\left(L^{2}+d_{1}^{2}\right)\left[\left(\sigma_{c o n}+E\right) \sqrt{d_{2}^{2}+L^{2}}-E \sqrt{\left(d_{2}+w_{p w}\right)^{2}+L^{2}}\right]}
$$

where

$$
\begin{aligned}
Z^{*}= & \frac{E\left(8 f_{p w}^{2} w_{p w}-8 f_{p w}^{2} d_{1}+3 w_{p w} d_{1}^{2}\right)}{3\left(L^{2}+d_{1}^{2}\right)^{3 / 2}}+\frac{\left(d_{1}+d_{2}\right)}{d_{2}}\left[\frac{\sigma_{c o n}-E}{\sqrt{L^{2}+d_{1}^{2}}}-\frac{8 E f_{p w}^{2}}{3\left(L^{2}+d_{1}^{2}\right)^{3 / 2}}+\frac{E \sqrt{L^{2}+\left(d_{1}-w_{p w}\right)^{2}}}{L^{2}+d_{1}^{2}}\right] \\
& +\frac{E d_{1} d_{2}\left[\sigma_{c o n} \sqrt{L^{2}+d_{1}^{2}}-E\left(\sqrt{L^{2}+\left(d_{1}-w_{p w}\right)^{2}}-\sqrt{L^{2}+d_{1}^{2}}+8 f_{p w}^{2} / 3 \sqrt{L^{2}+d_{1}^{2}}\right)\right]}{\left(L^{2}+d_{1}^{2}\right) \sqrt{L^{2}+d_{2}^{2}}}
\end{aligned}
$$

3.6. Structural Performance of PUSCs in the Condition Considering Both a Suction Wind Load and Vertical Loads. Considering that the effect of vertical loads may be either greater or lesser than that of a suction wind load, two cases are listed as follows.
(1) Case \#1: The Effect of the Vertical Loads Is Greater Than That of the Suction Wind Load. The vertical displacement of the inner strut, $w_{v s}$, and the local deformations of the ridge cables, $f_{v s}$, can be denoted as follows:

$$
\begin{aligned}
w_{v s}= & w_{v}-w_{s w} \\
= & \frac{L q_{v} / 2+E A_{r, v s} q_{v}^{2} L^{3} d_{1} / 24 H_{r 2, v s}^{2}\left(L^{2}+d_{1}^{2}\right)}{E A_{r, v s} q_{v}^{2} L^{3} / 24 H_{r 2, v s}^{2}\left(L^{2}+d_{1}^{2}\right)+E A_{r, v s} d_{1}^{2} /\left(L^{2}+d_{1}^{2}\right)^{3 / 2}+E A_{d, v s} d_{2}^{2} /\left(L^{2}+d_{2}^{2}\right)^{3 / 2}+H_{r 1, v s}\left(d_{1}+d_{2}\right) / L d_{2}} \\
& -\frac{L q_{s w} / 2+E A_{r, v s} q_{s w}^{2} L^{2} d_{1} / 24 H_{r 2}^{2} \sqrt{\left(L^{2}+d_{1}^{2}\right)}}{E A_{r, v s} q_{s w}^{2} L^{2} / 24 H_{r 2, v s}^{2} \sqrt{\left(L^{2}+d_{1}^{2}\right)}+E A_{r, v s} d_{1}^{2} /\left(L^{2}+d_{1}^{2}\right)^{3 / 2}+E A_{d, v s} d_{2}^{2} /\left(L^{2}+d_{2}^{2}\right)^{3 / 2}+H_{r 1, v s}\left(d_{1}+d_{2}\right) / L d_{2}} \\
f_{v s}= & f_{v}-\frac{L f_{s w}}{\sqrt{L^{2}+\left(d_{1}+w_{s w}\right)^{2}}} \approx \frac{q_{v} L^{2}-q_{s w} L \sqrt{L^{2}+d_{1}^{2}}}{8 H_{r 2, v s}}
\end{aligned}
$$




$$
\begin{aligned}
T_{d 2, v s}= & A_{d, v s} \sigma_{c o n}=T_{d 1, v s}+E A_{d, v s} \frac{\Delta L_{d, v s}}{L_{d, v s}}=\frac{H_{r 1, v s} d_{1} \sqrt{d_{2}^{2}+L^{2}}}{L d_{2}}+\frac{E A_{d, v s} \sqrt{\left(d_{2}+w_{v s}\right)^{2}+L^{2}}}{\sqrt{d_{2}^{2}+L^{2}}}-E A_{d, v s} \\
H_{r 1, v s} & =\sigma_{c o n} A_{r, v s} \frac{L}{\sqrt{L^{2}+d_{1}^{2}}}-E A_{r, v s} \frac{L \Delta l_{r, v s}}{L^{2}+d_{1}^{2}}=A_{r, v s}\left(\sigma_{c o n} \frac{L}{\sqrt{L^{2}+d_{1}^{2}}}-\frac{E L \Delta l_{r, v s}}{L^{2}+d_{1}^{2}}\right) \\
& =A_{r, v s} \frac{\left[\left(\sigma_{c o n}+E\right) L \sqrt{L^{2}+d_{1}^{2}}-E L \sqrt{L^{2}+\left(d_{1}-w_{v s}\right)^{2}}-8 E L f_{v s}^{2} / 3 \sqrt{L^{2}+d_{1}^{2}}\right]}{\left(L^{2}+d_{1}^{2}\right)} \\
A_{d, v s} & =\frac{A_{r, v s} d_{1}\left(L^{2}+d_{2}^{2}\right)\left[\left(\sigma_{c o n}+E\right) \sqrt{L^{2}+d_{1}^{2}}-E \sqrt{L^{2}+\left(d_{1}-w_{v s}\right)^{2}}-8 E f_{v s}^{2} / 3 L\right]}{d_{2}\left(L^{2}+d_{1}^{2}\right)\left[\left(\sigma_{c o n}+E\right) \sqrt{L^{2}+d_{2}^{2}}-E \sqrt{L^{2}+\left(d_{2}+w_{v s}\right)^{2}}\right]}
\end{aligned}
$$

In this case, the sectional areas of the ridge cables and diagonal cables in the condition considering both a suction wind load and vertical loads, $A_{r, v s}$ and $A_{d, v s}$, can be denoted by using (56), (57), and (60).

$$
\begin{aligned}
& A_{r, v s}=\frac{L\left(q_{v}-q_{s w}\right)}{2 B^{*}} \\
& A_{d, v s}=\frac{L d_{1}\left(q_{v}-q_{s w}\right)\left(L^{2}+d_{2}^{2}\right)\left[\sigma_{c o n} \sqrt{L^{2}+d_{1}^{2}}-E\left(\sqrt{L^{2}+\left(d_{1}-w_{v s}\right)^{2}}-\sqrt{L^{2}+d_{1}^{2}}+8 f_{v s}^{2} / 3 L\right)\right]}{2 B^{*} d_{2}\left(L^{2}+d_{1}^{2}\right)\left[\left(\sigma_{c o n}+E\right) \sqrt{d_{2}^{2}+L^{2}}-E \sqrt{\left(d_{2}+w_{v s}\right)^{2}+L^{2}}\right]}
\end{aligned}
$$

where

$$
\begin{aligned}
B^{*}= & \frac{w_{v s}\left(d_{1}+d_{2}\right)\left[L\left(\sigma_{c o n}+E\right) \sqrt{L^{2}+d_{1}^{2}}-E L \sqrt{L^{2}+\left(d_{1}-w_{v s}\right)^{2}}-8 E f_{v s}^{2} / 3\right]}{L d_{1} d_{2}\left(L^{2}+d_{2}^{2}\right)} \\
& +\frac{8 E L f_{v s}^{2}\left[L q_{v}^{2}\left(w_{v s}-d_{1}\right)+q_{p w}^{2} d_{1} \sqrt{L^{2}+d_{1}^{2}}\right]}{3\left(L^{2}+d_{1}^{2}\right)\left(q_{v} L-q_{p w} \sqrt{L^{2}+d_{1}^{2}}\right)^{2}} \\
& +\frac{E d_{1} d_{2} w_{v s}\left[\left(\sigma_{c o n}+E\right) \sqrt{L^{2}+d_{1}^{2}}-E \sqrt{L^{2}+\left(d_{1}-w_{v s}\right)^{2}}-8 E f_{v s}^{2} / 3 L\right]}{\left(L^{2}+d_{1}^{2}\right) \sqrt{L^{2}+d_{2}^{2}}\left[\left(\sigma_{c o n}+E\right) \sqrt{L^{2}+d_{2}^{2}}-E \sqrt{L^{2}+\left(d_{2}+w_{v s}\right)^{2}}\right]}
\end{aligned}
$$

(2) Case \#2: The effect of the Suction Wind Load Is Greater Than That of the Vertical Loads. The vertical displacement of the inner strut, $w_{v s}$, and the local deformations of the ridge cables, $f_{v s}$, can be denoted as follows: 


$$
\begin{aligned}
w_{v s}= & w_{s w}-w_{v} \\
= & \frac{L q_{s w} / 2+E A_{r, v s} q_{s w}^{2} L^{2} d_{1} / 24 H_{r 2}^{2} \sqrt{\left(L^{2}+d_{1}^{2}\right)}}{E A_{r, v s} q_{s w}^{2} L^{2} / 24 H_{r 2, v s}^{2} \sqrt{\left(L^{2}+d_{1}^{2}\right)}+E A_{r, v s} d_{1}^{2} /\left(L^{2}+d_{1}^{2}\right)^{3 / 2}+E A_{d, v s} d_{2}^{2} /\left(L^{2}+d_{2}^{2}\right)^{3 / 2}+H_{r 1, v s}\left(d_{1}+d_{2}\right) / L d_{2}} \\
& -\frac{L q_{v} / 2+E A_{r, v s} q_{v}^{2} L^{3} d_{1} / 24 H_{r 2, v s}^{2}\left(L^{2}+d_{1}^{2}\right)}{E A_{r, v s} q_{v}^{2} L^{3} / 24 H_{r 2, v s}^{2}\left(L^{2}+d_{1}^{2}\right)+E A_{r, v s} d_{1}^{2} /\left(L^{2}+d_{1}^{2}\right)^{3 / 2}+E A_{d, v s} d_{2}^{2} /\left(L^{2}+d_{2}^{2}\right)^{3 / 2}+H_{r 1, v s}\left(d_{1}+d_{2}\right) / L d_{2}} \\
f_{v s}= & f_{s w}-\frac{L f_{v}}{\sqrt{L^{2}+\left(d_{1}+w_{v}\right)^{2}}} \approx \frac{q_{s w} L \sqrt{L^{2}+d_{1}^{2}}}{8 H_{r 2, v s}}-\frac{q_{v} L^{3}}{8 H_{r 2, v s} \sqrt{L^{2}+d_{1}^{2}}}
\end{aligned}
$$

Assuming that all of the cables reach their design strength after loading, the following equations can be obtained:

$$
\begin{aligned}
T_{d 2, v s} & =A_{d, v s} \sigma_{c o n}=T_{d 1, v s}+E A_{d, v s} \frac{\Delta L_{d, v s}}{L_{d, v s}}=\frac{H_{r 1, v s} d_{1} \sqrt{d_{2}^{2}+L^{2}}}{L d_{2}}+\frac{E A_{d, v s} \sqrt{\left(d_{2}+w_{v}\right)^{2}+L^{2}}}{\sqrt{d_{2}^{2}+L^{2}}}-E A_{d, v s} \\
H_{r 1, v s} & =\sigma_{c o n} A_{r, v s} \frac{L}{\sqrt{L^{2}+d_{1}^{2}}}-E A_{r, v s} \frac{L \Delta l_{r, v s}}{L^{2}+d_{1}^{2}}=A_{r, v s}\left(\frac{\left.\sigma_{c o n} \frac{L}{\sqrt{L^{2}+d_{1}^{2}}}-\frac{E L \Delta l_{r, v s}}{L^{2}+d_{1}^{2}}\right)}{3\left(L^{2}+d_{1}^{2}\right)^{1.5}}-\frac{E L \sqrt{L^{2}+\left(d_{1}-w_{v s}\right)^{2}}}{L^{2}+d_{1}^{2}}\right) \\
& =A_{r, v s}\left(\frac{\left(\sigma_{c o n}+E\right) L}{\sqrt{L^{2}+d_{1}^{2}}}-\frac{8 E L f_{v s}^{2}}{3}\right) \\
A_{d, v s} & \left.=A_{r, v s} \frac{d_{1}\left(d_{2}^{2}+L^{2}\right)\left[\left(\sigma_{c o n}+E\right) \sqrt{L^{2}+d_{1}^{2}}-E \sqrt{L^{2}+\left(d_{1}-w_{v s}\right)^{2}}-8 E f_{v s}^{2} / 3 \sqrt{L^{2}+d_{1}^{2}}\right]}{d_{2}\left(L^{2}+d_{1}^{2}\right)\left[\left(\sigma_{c o n}+E\right) \sqrt{d_{2}^{2}+L^{2}}-E \sqrt{\left(d_{2}+w_{v s}\right.}\right)^{2}+L^{2}}\right]
\end{aligned}
$$

In this case, the sectional areas of the ridge cables and diagonal cables in the condition considering both a suction wind load and vertical loads, $A_{r, v s}$ and $A_{d, v s}$, can be denoted by using (64), (65), and (68).

$$
\begin{aligned}
& A_{r, v s}=\frac{L\left(q_{s w}-q_{v}\right)}{2 C^{*}} \\
& A_{d, v s}=\frac{L d_{1}\left(q_{s w}-q_{v}\right)\left(d_{2}^{2}+L^{2}\right)\left[\left(\sigma_{c o n}+E\right) \sqrt{L^{2}+d_{1}^{2}}-E \sqrt{L^{2}+\left(d_{1}-w_{v s}\right)^{2}}-8 E f_{v s}^{2} / 3 \sqrt{L^{2}+d_{1}^{2}}\right]}{2 C^{*} d_{2}\left(L^{2}+d_{1}^{2}\right)\left[\left(\sigma_{c o n}+E\right) \sqrt{d_{2}^{2}+L^{2}}-E \sqrt{\left(d_{2}+w_{v s}\right)^{2}+L^{2}}\right]}
\end{aligned}
$$




$$
\begin{aligned}
C^{*}= & \frac{w_{v s}\left(d_{1}+d_{2}\right)\left[L\left(\sigma_{c o n}+E\right) \sqrt{L^{2}+d_{1}^{2}}-E L \sqrt{L^{2}+\left(d_{1}-w_{v s}\right)^{2}}-8 E f_{v s}^{2} / 3\right]}{L d_{2}\left(L^{2}+d_{2}^{2}\right)} \\
& +\frac{E d_{1} d_{2} w_{v s}\left[\left(\sigma_{c o n}+E\right) \sqrt{L^{2}+d_{1}^{2}}-E \sqrt{L^{2}+\left(d_{1}-w_{v s}\right)^{2}}-8 E f_{v s}^{2} / 3 L\right]}{\left(L^{2}+d_{1}^{2}\right) \sqrt{L^{2}+d_{2}^{2}}\left[\left(\sigma_{c o n}+E\right) \sqrt{L^{2}+d_{2}^{2}}-E \sqrt{L^{2}+\left(d_{2}+w_{v s}\right)^{2}}\right]} \\
& +\frac{8 E f_{v s}^{2}\left[q_{s w}^{2} d_{1} \sqrt{L^{2}+d_{1}^{2}}+L q_{v}^{2}\left(w_{v s}-d_{1}\right)\right]}{3\left(q_{s w}\left(L^{2}+d_{1}^{2}\right)-q_{v} L^{2}\right)^{2}}+\frac{E w_{v s} d_{1}^{2}}{\left(L^{2}+d_{1}^{2}\right)^{3 / 2}}
\end{aligned}
$$

3.7. Structural Performance of PUSCs in the Condition Considering Both a Pressure Wind Load and Vertical Loads. Adding the effect of both the pressure wind load and the vertical loads, the vertical displacement of the inner strut, $w_{v p}$, and the local deformations of the ridge cables, $f_{v p}$, can be denoted as follows:

$$
\begin{aligned}
w_{v p}= & w_{v}+w_{p w} \\
= & \frac{L q_{v} / 2+E A_{r, v p} q_{v}^{2} L^{3} d_{1} / 24 H_{r 2, v p}^{2}\left(L^{2}+d_{1}^{2}\right)}{E A_{r, v p} q_{v}^{2} L^{3} / 24 H_{r 2, v p}^{2}\left(L^{2}+d_{1}^{2}\right)+E A_{r, v p} d_{1}^{2} /\left(L^{2}+d_{1}^{2}\right)^{3 / 2}+E A_{d, v p} d_{2}^{2} /\left(L^{2}+d_{2}^{2}\right)^{3 / 2}+H_{r 1, v p}\left(d_{1}+d_{2}\right) / L d_{2}} \\
& +\frac{L q_{p w} / 2+E A_{r, v p} q_{p w}^{2} L^{2} d_{1} / 24 H_{r 2}^{2} \sqrt{\left(L^{2}+d_{1}^{2}\right)}}{E A_{r, v p} q_{p w}^{2} L^{2} / 24 H_{r 2, v p}^{2} \sqrt{\left(L^{2}+d_{1}^{2}\right)}+E A_{r, v p} d_{1}^{2} /\left(L^{2}+d_{1}^{2}\right)^{3 / 2}+E A_{d, v p} d_{2}^{2} /\left(L^{2}+d_{2}^{2}\right)^{3 / 2}+H_{r 1, v p}\left(d_{1}+d_{2}\right) / L d_{2}} \\
f_{v p}= & f_{v}+\frac{L f_{p w}}{\sqrt{L^{2}+\left(d_{1}+w_{p w}\right)^{2}}} \approx \frac{q_{v} L^{2}+q_{p w} L \sqrt{L^{2}+d_{1}^{2}}}{8 H_{r 2, v p}}
\end{aligned}
$$

Assuming that all of the cables reach their design strength after loading, the following equations can be obtained:

$$
\begin{aligned}
T_{d 2, v p} & =A_{d, v p} \sigma_{c o n}=T_{d 1, v p}+E A_{d, v p} \frac{\Delta L_{d, v p}}{L_{d, v p}}=\frac{H_{r 1, v p} d_{1} \sqrt{d_{2}^{2}+L^{2}}}{L d_{2}}+\frac{E A_{d, v p} \sqrt{\left(d_{2}+w_{v}\right)^{2}+L^{2}}}{\sqrt{d_{2}^{2}+L^{2}}}-E A_{d, v p} \\
H_{r 1, v p} & =\sigma_{c o n} A_{r, v p} \frac{L}{\sqrt{L^{2}+d_{1}^{2}}}-E A_{r, v p} \frac{L \Delta l_{r, v p}}{L^{2}+d_{1}^{2}}=A_{r, v p}\left(\frac{\left.\sigma_{c o n} \frac{L}{\sqrt{L^{2}+d_{1}^{2}}}-\frac{E L \Delta l_{r, v p}}{L^{2}+d_{1}^{2}}\right)}{3\left(L^{2}+d_{1}^{2}\right)^{3 / 2}}-\frac{E \sqrt{L^{2}+\left(d_{1}-w_{v p}\right)^{2}}}{L^{2}+d_{1}^{2}}\right) \\
& =A_{r, v p}\left(\frac{\left(\sigma_{c o n}+E\right) L}{\sqrt{L^{2}+d_{1}^{2}}}-\frac{8 E L f_{v p}^{2}}{3\left(d^{2}+L^{2}\right)\left[\left(\sigma_{c o n}+E\right) \sqrt{L^{2}+d_{1}^{2}}-E \sqrt{L^{2}+\left(d_{1}-w_{v p}\right)^{2}}-8 E f_{v p}^{2} / 3 \sqrt{L^{2}+d_{1}^{2}}\right]}\right. \\
A_{d, v p} & =A_{r, v p} \frac{d_{1}\left(L^{2}+d_{1}^{2}\right)\left[\left(\sigma_{c o n}+E\right) \sqrt{d_{2}^{2}+L^{2}}-E \sqrt{\left(d_{2}+w_{v p}\right)^{2}+L^{2}}\right]}{\left(d_{2}\right]}
\end{aligned}
$$


In this case, the sectional areas of the ridge cables and diagonal cables in the condition considering both a pressure wind load and vertical loads, $A_{r, v p}$ and $A_{d, v p}$, can be denoted by using (72), (73), and (76).

$$
\begin{aligned}
& A_{r, v p}=\frac{L\left(q_{p w}+q_{v}\right)}{2 D^{*}} \\
& A_{d, v p}=\frac{L d_{1}\left(q_{p w}+q_{v}\right)\left(d_{2}^{2}+L^{2}\right)\left[\left(\sigma_{c o n}+E\right) \sqrt{L^{2}+d_{1}^{2}}-E \sqrt{L^{2}+\left(d_{1}-w_{v p}\right)^{2}}-8 E f_{v p}^{2} / 3 \sqrt{L^{2}+d_{1}^{2}}\right]}{2 D^{*} d_{2}\left(L^{2}+d_{1}^{2}\right)\left[\left(\sigma_{c o n}+E\right) \sqrt{d_{2}^{2}+L^{2}}-E \sqrt{\left(d_{2}+w_{v p}\right)^{2}+L^{2}}\right]}
\end{aligned}
$$

where

$$
\begin{aligned}
D^{*}= & \frac{w_{v p}\left(d_{1}+d_{2}\right)\left[L\left(\sigma_{c o n}+E\right) \sqrt{L^{2}+d_{1}^{2}}-E L \sqrt{L^{2}+\left(d_{1}-w_{v p}\right)^{2}}-8 E f_{v p}^{2} / 3\right]}{L d_{2}\left(L^{2}+d_{2}^{2}\right)} \\
& +\frac{E d_{1} d_{2} w_{v p}\left[\left(\sigma_{c o n}+E\right) \sqrt{L^{2}+d_{1}^{2}}-E \sqrt{L^{2}+\left(d_{1}-w_{v p}\right)^{2}}-8 E f_{v p}^{2} / 3 \sqrt{L^{2}+d_{1}^{2}}\right]}{\left(L^{2}+d_{1}^{2}\right) \sqrt{L^{2}+d_{2}^{2}}\left[\left(\sigma_{c o n}+E\right) \sqrt{L^{2}+d_{2}^{2}}-E \sqrt{L^{2}+\left(w_{v p}+d_{2}\right)^{2}}\right]} \\
& +\frac{8 E f_{v p}^{2}\left[q_{p w}^{2} \sqrt{L^{2}+d_{1}^{2}}\left(w_{v p}-d_{1}\right)-q_{v}^{2} L d_{1}\right]}{3\left(L^{2}+d_{1}^{2}\right)\left(q_{p w} \sqrt{L^{2}+d_{1}^{2}}+q_{v} L\right)^{2}}+\frac{E w_{v p} d_{1}^{2}}{\left(L^{2}+d_{1}^{2}\right)^{3 / 2}}
\end{aligned}
$$

3.8. Design of Other Structural Parameters. Generally, the forms of the external load added on a PUSC can be divided into three types, as follows:

Type (a): only vertical loads, as discussed in Section 3.3;

Type (b): vertical loads with a suction wind load, as discussed in Section 3.6;

Type (c): vertical loads with a pressure wind load, as discussed in Section 3.7.

After the form of the external load is determined, the values of $H_{r 1}, A_{r}$, and $A_{d}$ are defined as follows:

$$
\begin{array}{r}
H_{r 1}= \begin{cases}H_{r 1, v} & \text { for Type }(a) \\
H_{r 1, v s} & \text { for Type }(b) \\
H_{r 1, v p} & \text { for Type }(c)\end{cases} \\
A_{r}= \begin{cases}A_{r, v} & \text { for Type }(a) \\
A_{r, v s} & \text { for Type (b) } \\
A_{r, v p} & \text { for Type (c) }\end{cases}
\end{array}
$$

$$
\begin{gathered}
A_{d}= \begin{cases}A_{d, v} & \text { for Type }(a) \\
A_{d, v s} & \text { for Type }(b) \\
A_{d, v p} & \text { for Type }(c)\end{cases} \\
w= \begin{cases}w_{v} & \text { for Type }(a) \\
w_{v s} & \text { for Type }(b) \\
w_{v p} & \text { for Type }(c)\end{cases} \\
f= \begin{cases}f_{v} & \text { for Type }(a) \\
f_{v s} & \text { for Type }(b) \\
f_{v p} & \text { for Type }(c) .\end{cases}
\end{gathered}
$$

The forces of the cables and inner struts in the initial prestress state can be calculated as

$$
T_{r 1}=\frac{H_{r 1} \sqrt{d_{1}^{2}+L^{2}}}{L}
$$




$$
\begin{aligned}
& T_{d 1}=\frac{H_{r 1} d_{1} \sqrt{d_{2}^{2}+L^{2}}}{L d_{2}} \\
& T_{s 1}=\frac{2 d_{1}}{\sqrt{L^{2}+d_{1}^{2}}} T_{r 1}
\end{aligned}
$$

The compression force of the inner struts in the loading condition is

$$
T_{s 2} \approx 2 T_{d 2} \frac{d_{2}+w}{\sqrt{L^{2}+\left(d_{2}+w\right)^{2}}}=\frac{2 A_{d} \sigma_{c o n}\left(d_{2}+w\right)}{\sqrt{L^{2}+\left(d_{2}+w\right)^{2}}}
$$

The relevant provision in [20] indicates that, for the inner struts that bear axial compressive forces, the buckling load should follow (84).

$$
T_{s 2}=N \leq \varphi A_{s} \sigma_{y}
$$

where $\varphi$ decreases with an increase in the slenderness ratio of the inner struts.

After obtaining the sectional areas of all the components, the weight of all the cables, the weight of the inner struts, and the total weight of a PUSC can be calculated as follows:

$$
\begin{aligned}
W_{\text {cable }} & =2 n \rho_{c} g\left(A_{r} \sqrt{L^{2}+d_{1}^{2}}+A_{d} \sqrt{L^{2}+d_{2}^{2}}\right) \\
W_{\text {strut }} & =n \rho_{s} g A_{s}\left(d_{1}+d_{2}\right) \\
W & =W_{\text {cable }}+W_{\text {strut }}
\end{aligned}
$$

The initial prestress forces and the total weight of the components can be obtained by determining the values of $A_{r}$, $A_{d}, d_{1}$, and $d_{2}$. Moreover, if the values of $L, q_{\mathrm{v}}, q_{s w}, q_{p w}, f$, $w$, and $\sigma_{c o n}$ are given, the optimum values of $A_{r}$ and $A_{d}$ are related to only $d_{1}$ and $d_{2}$.

\section{Structural Design of the PUSCs}

4.1. Design Objective. During the structural optimization design, the coordinates of the cable nodes and strut nodes are changed, the sectional areas of the cables and struts are changed, and the weights of the components are changed correspondingly. To resist external loads, a feasible PUSC design should possess a geometric configuration with sufficient sectional areas of cables and struts to supply an adequate overall stiffness and to form enough reaction forces. However, the excessive addition of lengths and sectional areas of the components will greatly increase the external load of the structure, which leads to larger component sectional areas, underutilization of material, and increased project costs. In this case, the minimum total weight of the cables and inner struts is taken as the design objective.

4.2. Design Variables. The coordinates of the cable nodes and strut nodes are important parameters that determine the geometric configuration of the PUSCs. Because the roof of a building must cover a specific area, $d_{1}$ and $d_{2}$, which determine the $z$-coordinates of the two nodes of the inner struts, are the only design variables in most cases. Furthermore, under some given boundary conditions, the magnitude and distribution of the prestress strongly affect the geometric configuration and the sectional areas of the cables and struts and thus determine the overall stiffness and total weight of the structure to some extent. However, as discussed above, the proper design of the forces and sectional areas of the cables can be obtained by determining the values of $d_{1}$ and $d_{2}$. The specifications of the inner struts can be selected afterwards. Therefore, $d_{1}$ and $d_{2}$ are the two design variables in this study.

4.3. Design Process. The detailed steps of the structural design are as follows:

(1) Preparation: Determine the values of $n, L, E, \sigma_{\text {con }}$, $q_{s w}\left(\right.$ or $\left.q_{p w}\right), \rho_{c}$, and $\rho_{s}$ and the variation ranges of $d_{1}$ and $d_{2}$. Determine the initial uniform vertical line load, $q_{v}(0)$ (without considering the self-weight of the cables and struts), the limited local displacement of the ridge cables, $f_{\text {con }}$, the limited mid-span displacement of the entire structure, $w_{\text {con }}$, and the permissible variation in $W, \Delta W_{\text {lim }}$. Separate the values of $d_{1}$ and $d_{2}$ into $n$ groups (i.e., $\left(d_{1,1}, d_{2,1}\right),\left(d_{1,2}, d_{2,2}\right)$, $\left(d_{1,3}, d_{2,3}\right), \ldots,\left(d_{1, i}, d_{2, i}\right), \ldots$, and $\left.\left(d_{1, n}, d_{2, n}\right)\right)$, and assign them the names group- 1 , group- $2, \ldots$, group- $i, \ldots$, and group- $n$, respectively.

(2) Type determination: according to the external loads added on the PUSC, select the type of loading condition, as listed in Section 3.8. If the loading condition belongs to Type (a) or Type (c), start the next step. However, if the loading condition belongs to Type (b), two cases should be compared first. Calculate the values of $w_{v s}$ by using both (56) and (64), and choose the positive one as the correct value; then, select the corresponding case as the correct case of Type (b). For example, if $w_{v s}$ calculated by (56) is positive, case \#1 is the correct case for the given loading condition. Then, start the next step.

(3) Iterative solution for group- $i$

(a) 1st iteration: define $q_{v}=q_{v}(0), d_{1}=d_{1, i}, d_{2}=d_{2, i}, f=$ $f_{\text {con }}$, and $w=w_{\text {con }}$. Then, determine the structural parameters as follows:

(1) For Type (a): calculate the values of $A_{r}$ and $A_{d}$ by (44), (45), (46), and (80), and define $A_{r i}(0)=A_{r}$ and $A_{d i}(0)=A_{d}$.

(2) For Type (b), case \#1: calculate the values of $A_{r}$ and $A_{d}$ by (61), (62), (63), and (80), and define $A_{r i}(0)=A_{r}$ and $A_{d i}(0)=$ $A_{d}$.

(3) For Type (b), case \#2: calculate the values of $A_{r}$ and $A_{d}$ by (69), (70), (71), and (80), and define $A_{r i}(0)=A_{r}$ and $A_{d i}(0)=A_{d}$.

(4) For Type (c): calculate the values of $A_{r}$ and $A_{d}$ by (77), (78), (79), and (80), and define $A_{r i}(0)=A_{r}$ and $A_{d i}(0)=A_{d}$.

Then, calculate the sectional area of the inner struts and the self-weight of all the cables and struts by (84), (85), and $(88)$ and define them as $A_{s i}(0)$ and $W_{i}(0)$, respectively. Add $W_{i}(0)$ to the total vertical load, as shown in (89). Subsequently, define $q_{v}=q_{v}(1)$, and determine the sectional areas of the ridge cables and the diagonal cables, $A_{r i}(1)$ and $A_{d i}(1)$, by using the same process of determining $A_{r i}(0)$ and $A_{d i}(0)$. Calculate the sectional area of the inner struts and 
the weight of all the cables and struts, $A_{s i}(1)$ and $W_{i}(1)$, respectively, by (84), (85), and (88). Check the accuracy criterion of $\Delta W_{i}(1)$ by (90).

$$
\begin{gathered}
q_{v}(1)=q_{v}(0)+\frac{W_{i}(0) g}{\pi\left(L^{2}+d_{1, i}^{2}\right)} \\
\Delta W_{i}(1)=\left|W_{i}(1)-W_{i}(0)\right| \leq \Delta W_{l i m}
\end{gathered}
$$

If (90) is satisfied, the iterative solution is completed. If not, start the next iteration.

(c) $k$ th iteration $(k \geq 2)$ : add the self-weight of the cables and struts from the $(k-1)^{\text {th }}$ iteration to the total vertical load, as shown in (91). Define $q_{v}=q_{v}(k)$; calculate the sectional areas of the ridge cables and the diagonal cables as $A_{r i}(k)$ and $A_{d i}(k)$, by the same step of determining $A_{r i}(0)$ and $A_{d i}(0)$. Obtain the sectional area of the inner struts and the self-weight of all the cables and struts as $A_{s i}(k)$ and $W_{i}(k)$, respectively, by (84), (85), and (88). Check the accuracy criterion of $\Delta W_{i}(k)$ by (92).

$$
\begin{gathered}
q_{v}(k)=q_{v}(0)+\frac{w_{i}(k-1) g}{\pi\left(L^{2}+d_{1, i}^{2}\right)} \\
\Delta W_{i}(k)=\left|W_{i}(k)-W_{i}(k-1)\right| \leq \Delta W_{\text {lim }}
\end{gathered}
$$

If (92) is satisfied, the iterative solution is completed. If not, start the next iteration.

Assuming that $\Delta W_{i}(m)$ meets the accuracy criterion, for the required sectional area of the ridge cables of group- $i, A_{r i}$ $=A_{r i}(m)$, the required sectional area of the diagonal cables of group- $i, A_{d i}=A_{d i}(m)$, the axial force of the inner struts of group- $i, T_{s 2, i}=T_{s 2, i}(m)$, and the total self-weight of all the cables and struts of group- $i, W_{i}=W_{i}(m)$. Moreover, the initial forces of the ridge cables and the diagonal cables of group- $i, T_{r 1, i}$ and $T_{d 1, i}$ can then be obtained using (10) and (11), respectively. Then, export these structural parameters.

(3) Final computation: after completing the analyses of all $n$ groups, compare the total weights of the cables and struts, calculated for each group, choose the minimum value as the rational mode (i.e., $W_{\text {rational }}$ ), and define the structural parameters, corresponding to the rational mode, as the required parameters.

$$
W_{\text {rational }}=\min \left\{W_{1}, W_{2}, \ldots, W_{n}\right\}
$$

The detailed process is shown in Figure 4.

\section{Example}

5.1. Description of a Typical PUSC Project. The typical PUSC project chosen for this study is the roof of the Shijiazhuang International Exhibition Centre in Hebei Province, China. The length of the longitudinal span is $137.5 \mathrm{~m}$, and the length of the horizontal span is $72 \mathrm{~m}$; 10 pieces are used.

The cables have an elastic modulus of $1.95 \times 10^{5} \mathrm{MPa}$, a design strength $\left(\sigma_{\text {con }}\right)$ of $928 \mathrm{MPa}$, and a density of $7.85 \times$ $10^{3} \mathrm{~kg} / \mathrm{m}^{3}$. The inner strut has an elastic modulus of $2.06 \times$ $10^{5} \mathrm{MPa}$, a yield strength $\left(\sigma_{y}\right)$ of $310 \mathrm{MPa}$, and a density of $7.85 \times 10^{3} \mathrm{~kg} / \mathrm{m}^{3}$.
TABLE 1: Loading combinations.

\begin{tabular}{lc}
\hline Name & Loading combination \\
\hline LC-1 & $1.2 D^{\prime}+1.4 L^{\prime}+1.0 P^{\prime}$ \\
LC-2 & $1.0 D^{\prime}+1.4 W^{\prime}+1.0 P^{\prime}$ \\
LC-3 & $1.0 D^{\prime}-1.4 W^{\prime}+1.0 P^{\prime}$ \\
\hline
\end{tabular}

5.2. Design Loads and Loading Combinations. In this study, the structural design of a PUSC is evaluated under the combined effect of the following loads:

(1) The self-weight of the PUSC $\left(D^{\prime}\right)$, which includes the weight of all the structural components and the roofing system. The total weight of all the cables and the inner struts, $W$, is calculated after each iteration and is added to the structural weight. The weight of the roofing system is assumed to be $0.10 \mathrm{kN} / \mathrm{m}^{2}$.

(2) The roof live load $\left(L^{\prime}\right)$, which is equivalent to $0.50 \mathrm{kN} / \mathrm{m}^{2}$.

(3) The wind load $\left(W^{\prime}\right)$, which is added perpendicular to the ridge cables. According to [21], the basic wind pressure, $w_{0}$, is defined as $0.50 \mathrm{kN} / \mathrm{m}^{2}$, the surface roughness is chosen as $B$, the shape coefficient, $\mu_{s}$, is defined as 0.8 , the wind vibration coefficient, $\beta_{z}$, is selected as 1.8 , the wind-pressure height coefficient is $\mu_{z}=1.25$, and the wind load is $w_{k}=$ $\beta_{z} \mu_{s} \mu_{z} w_{0}=0.9 \mathrm{kN} / \mathrm{m}^{2}$.

(4) The prestress forces $\left(P^{\prime}\right)$, which are obtained from the results.

The combinations of these loads are divided into three cases, as listed in Table 1, where LC-1 considers the effects of $D^{\prime}, L^{\prime}$, and $P^{\prime}$ only, and LC-2 and LC-3 additionally consider $W^{\prime}$ to investigate the effect of both the vertical loads and the wind loads; among them, $+1.4 W^{\prime}$ and $-1.4 W^{\prime}$ denote adding and subtracting 1.4 times the wind suction load and wind pressure to the ridge cables, respectively.

5.3. Design Constraints. The following design constraints, related to the stresses in the cables, the vertical displacements of the inner strut nodes, the local deformations of the ridge cables, and the variations in vector height and sag height, are considered in the structural design process.

(a) Cable Stress. The maximum stress of all the cables should not exceed their design strength, which is $928 \mathrm{MPa}$.

(b) Vertical Displacements of the Inner Struts. The vertical displacement of the inner struts, $w$, must be restricted to $[0, L / 500]$ (i.e., $w_{\text {con }}=L / 500$ ).

(c) Local Deformation of the Ridge Cables. The maximum local deformation of the ridge cables, $f$, is restricted to $\left[0, \sqrt{L^{2}+d_{1}^{2}} / 200\right]$ (i.e., $\left.f_{\text {con }}=\sqrt{L^{2}+d_{1}^{2}} / 200\right)$.

(d) Variations in the Vector Height and the Sag Height. The vector height, $d_{1}$, and the sag height, $d_{2}$, both vary in the range of $[1000,36000] \mathrm{mm}$. 


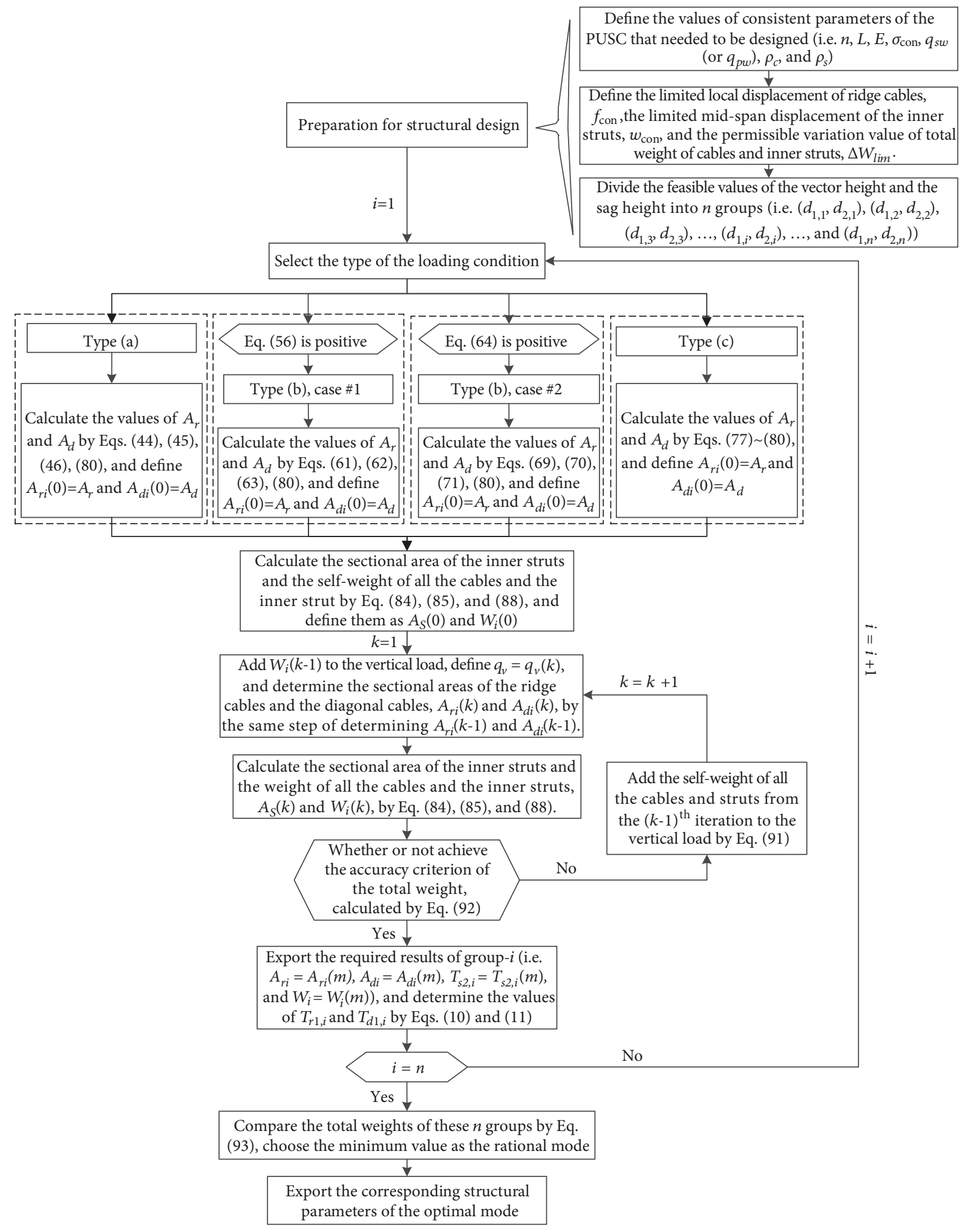

FIGURE 4: Structural design process for PUSCs. 
TABLE 2: Rational modes for the three loading combinations.

\begin{tabular}{lccc}
\hline Load case & LC-1 & LC-2 & LC-3 \\
\hline$d_{1}(\mathrm{~mm})$ & 25000 & 27000 & 28000 \\
$d_{2}(\mathrm{~mm})$ & 27000 & 29000 & 30000 \\
$A_{r}\left(\mathrm{~mm}^{2}\right)$ & 791.2 & 974.8 & 1165.8 \\
$A_{d}\left(\mathrm{~mm}^{2}\right)$ & 1111.75 & 1393.6 & 1676.7 \\
Dimensions of the inner strut & $260 \times 16$ & $275 \times 20$ & $305 \times 22$ \\
$(\mathrm{~mm} \times \mathrm{mm})$ & & & 1042.3 \\
$T_{r 1}(\mathrm{kN})$ & 866.1 & 999.6 & 1286.9 \\
$T_{d 1}(\mathrm{kN})$ & 823.4 & 2396.9 & 1234.1 \\
$W(\mathrm{kN})$ & 1822.9 & & 2959.2 \\
\hline
\end{tabular}

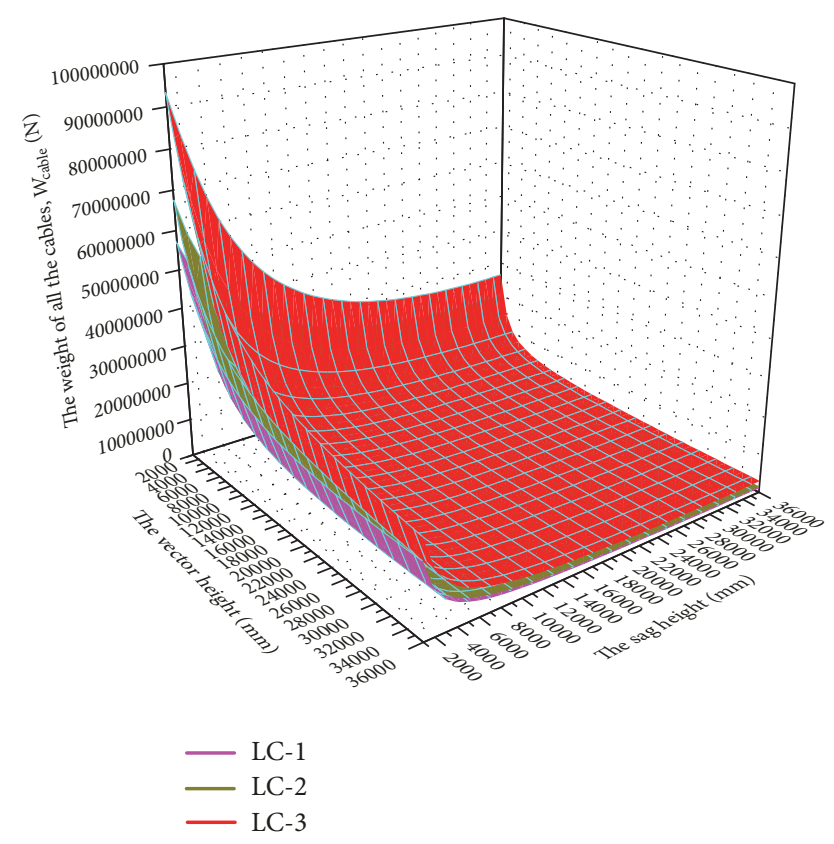

FIGURE 5: Three-dimensional contour maps for the weight of the cables, $W_{\text {cable }}$, for the three loading combinations.

5.4. Analysis Results and Discussion. The resultant forces of these three loading combinations are reduced in the following order: LC-3 > LC-2 > LC-1. Among them, the resultant force of LC-2 is directed upwards, while the other two are directed downwards.

The three-dimensional and two-dimensional contour maps of the weight of the cables, $W_{\text {cable }}$, for these three loading combinations are illustrated in Figures 5 and 6 , and the contour maps of the weight of the inner struts, $W_{\text {strut }}$ for these three loading combinations are illustrated in Figures 7 and 8, respectively. The contour maps display similar shapes (except for the maximum and minimum values), i.e., the variations in $W_{\text {cable }}$ and $W_{\text {strut }}$ for these three loading combinations can be summarized by the same law without considering the direction of the resultant forces. Furthermore, the lowest values of the contour maps are directly related to the size of the external load. As the external load increases, the lowest value of a contour map increases. As $d_{1}$ and $d_{2}$ increase, the geometric stiffness of the structure increases with the rise-tospan and sag-to-span ratios; thus, the component stiffness, which is formed by the sectional stiffness of the members, could be reduced. In this case, the sectional areas of cables correspondingly decrease, which leads to a decrease in the total weight of the cables and the initial pretension forces in the cables. In contrast, with increasing $d_{1}$ and $d_{2}$, the slenderness ratio of the inner struts also increases, resulting in an increase in the sectional area of the inner struts, as indicated in (85), greatly increasing the weight of the inner struts.

The contour maps of the total weight of the entire structure, $W$, for these three loading combinations are shown in Figures 9 and 10. Since the main contribution the total weight is from the cables, the contour maps of $W$ are analogous to those of $W_{\text {cable }}$. Due to the low geometric stiffness and the high pretension forces on the cables, the sectional areas of the cables are large for low values of $d_{1}$ and $d_{2}$, which leads to a high $W$ during the initial stage. As $d_{1}$ and $d_{2}$ increase, $W$ decreases, the geometric stiffness increases, the prestress decreases, and the sectional areas of the cables decrease. However, after reaching the minimum $W$, the weight of the inner struts (i.e., $W_{\text {strut }}$ ) significantly increases, increasing $W$ again. In this case, the rational mode of the PUSC for a specific loading combination shown in in Figures 9 and 10 is the corresponding combination of $d_{1}$ and $d_{2}$ at the lowest point that results in a suitable slenderness ratio of the inner strut, an appropriate prestress of the entire structure, and a minimum value of $W$.

For comparison, the rational structural modes for the three loading combinations employed in this paper are displayed in Table 2. All the parameters of the rational modes listed in Table 2 show that LC-3 > LC- $2>$ LC- 1 , which is the same as the sequence of the resultant forces of these loading combinations. Therefore, the external loads added on the structure greatly affect the design results of PUSCs. Considerable loads, added on a PUSC, require a large vector height (i.e., $d_{1}$ ) and a large sag height (i.e., $d_{2}$ ) to increase the overall stiffness; thus, the initial prestress forces of the components, the sectional areas of the cables, the dimensions of the inner struts, and the total weight of all the cables and struts are relatively large.

Furthermore, the loading direction is also an important factor for the structural response of PUSCs. A suction wind 


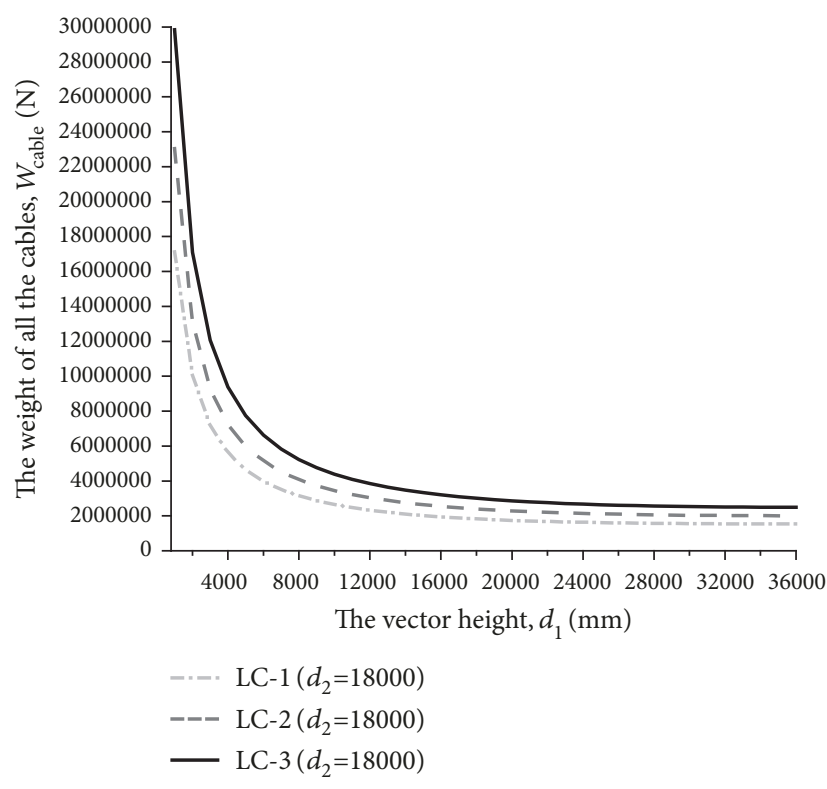

(a)

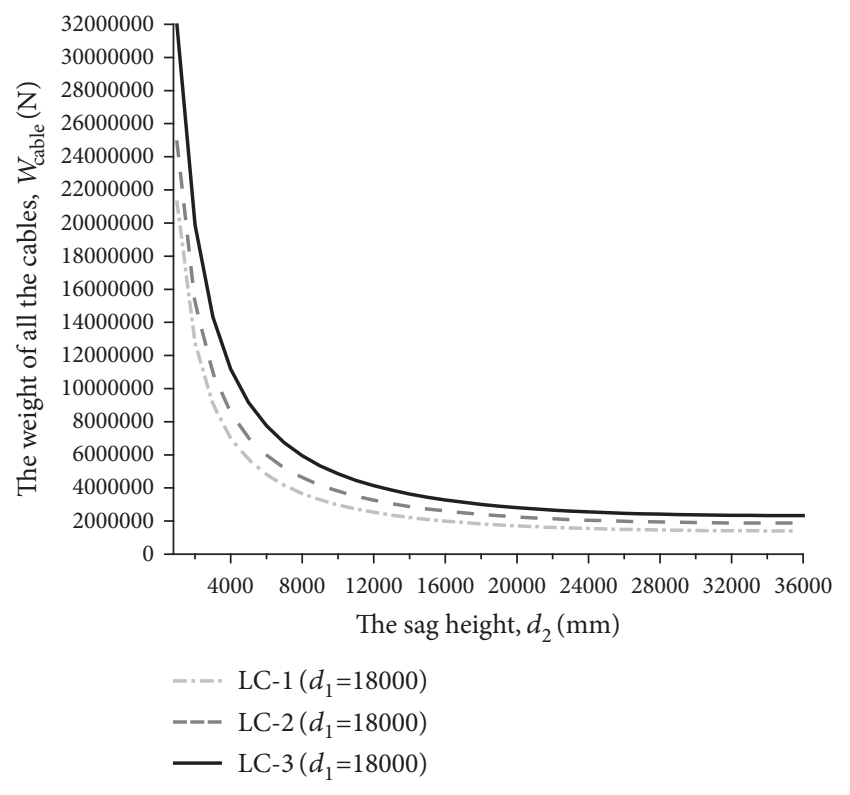

(b)

Figure 6: Two-dimensional contour maps for the weight of the cables, $W_{\text {cable, }}$, for the three loading combinations. (a) Variation in $W_{\text {cable }}$ with various values of $d_{1}\left(1000 \leq d_{1} \leq 360000\right)$. (b) Variation in $W_{\text {cable }}$ with various values of $d_{2}\left(1000 \leq d_{2} \leq 360000\right)$.

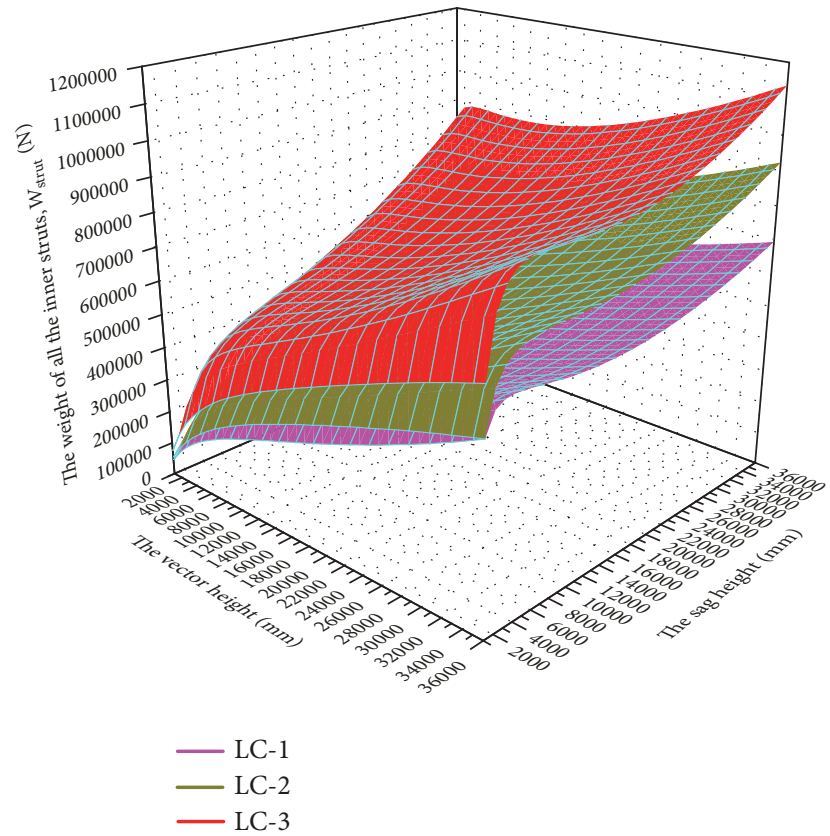

Figure 7: Three-dimensional contour maps for the weight of the inner struts, $W_{\text {strut }}$, for the three loading combinations.

load will offset part of the effect of vertical loads, reducing the local deformations of the ridge cables and the vertical deformations of the inner strut, whereas a pressure wind load would expand the forces added on the structure and thus increase the structural response.

Variations in $W$ with various values of $d_{1}$ and $d_{2}$ for the rational modes are displayed in Figure 11. The results show that the variation in $W$ exhibits three stages under external loads. For a consistent value of $d_{2}$ (or $d_{1}$ ), $W$ decreases sharply from a relatively high value during the initial increasing stage of $d_{1}$ (or $d_{2}$ ) and then starts to decrease gradually in the second stage. After reaching the minimum value, $W$ slowly increases during the last stage.

As shown in Table 2, the rational mode of LC-3 provides the PUSC with a greater vector height, sag height, cable sectional areas, inner strut dimensions, and prestress forces; thus, this mode could provide a greater geometric stiffness and prestress, allowing the PUCS to withstand additional external loads. Therefore, the rational mode of LC-3 is selected as the optimal designed mode to meet the requirements of all three loading combinations.

\section{Conclusions}

In this paper, a new theoretical method for the modelling and structural design of a PUSC was proposed. This method takes the vector height and the sag height as the design variables and the minimal weight of the entire structure as the design objective. According to the principle of stationary potential energy, the relations among the sectional areas of the cables, the prestress forces, the vector height, the sag height, the overall displacement, and the local deformation were proposed. The static responses of the PUSC under vertical loads and wind loads were obtained afterwards. Subsequently, a detailed description of the design procedure was introduced to obtain the sectional areas of the cables, the specifications of the inner strut, and the prestress forces of these components by giving only the variation ranges of the vector height and the sag height. Finally, a calculation model of a $100 \mathrm{~m}$-span PUSC was built and optimized to verify the 

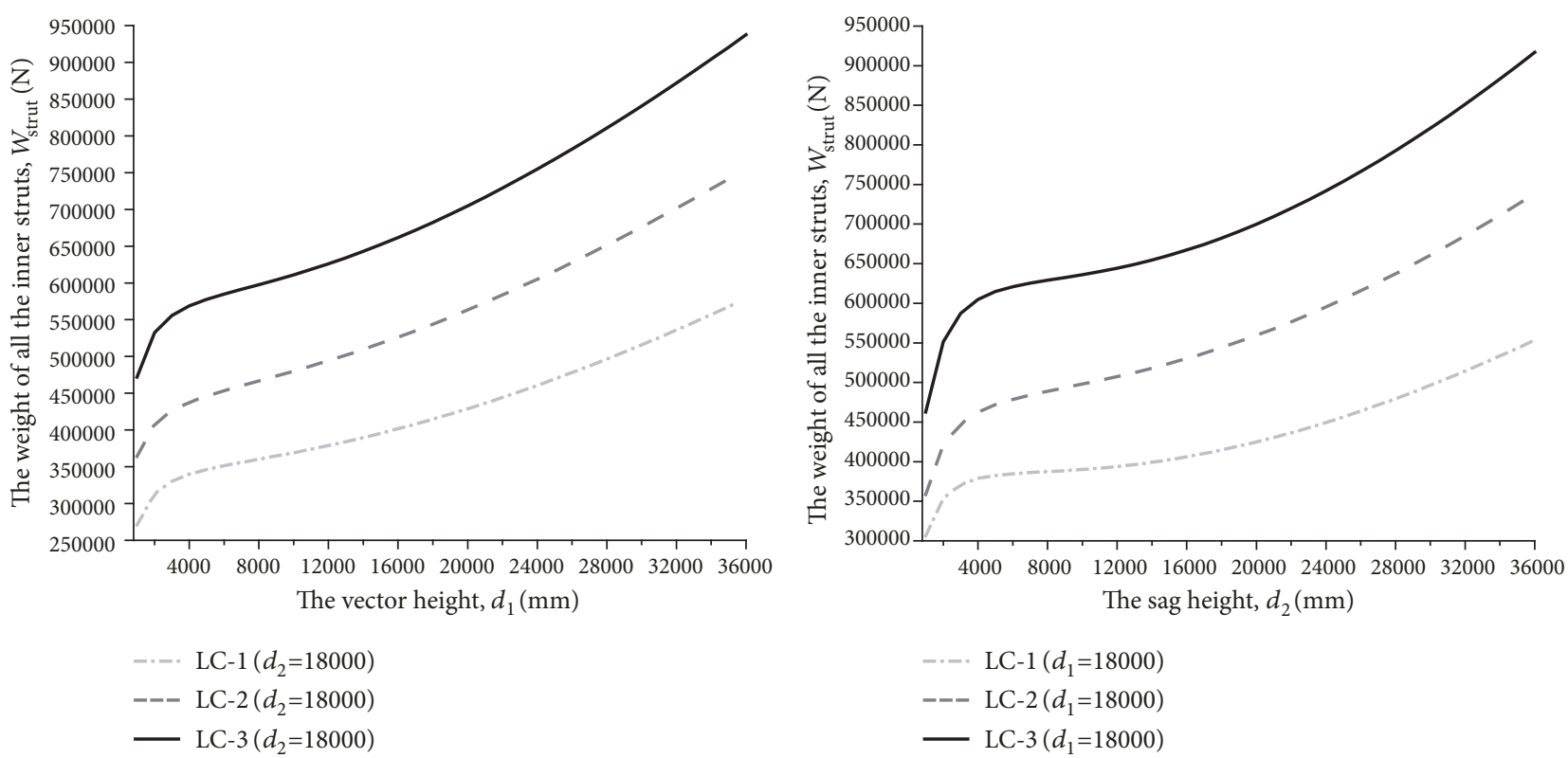

(a)

(b)

FIGURE 8: Two-dimensional contour maps for the weight of the inner struts, $W_{\text {strut }}$, for the three loading combinations. (a) Variation in $W_{\text {cable }}$ with various values of $d_{1}\left(1000 \leq d_{1} \leq 360000\right)$. (b) Variation in $W_{\text {cable }}$ with various values of $d_{2}\left(1000 \leq d_{2} \leq 360000\right)$.

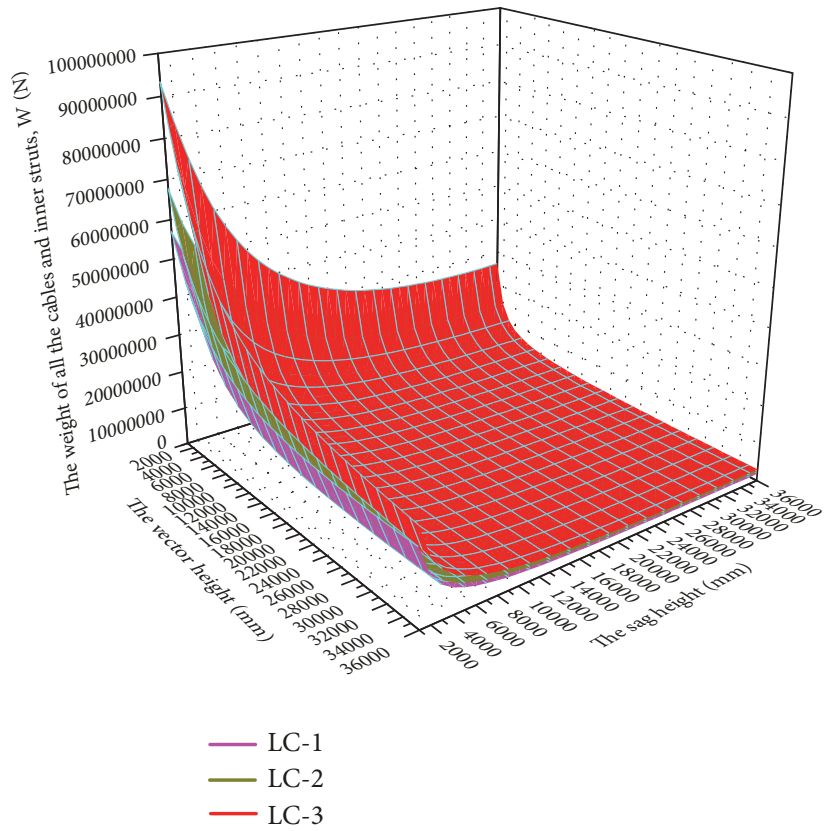

FIgURE 9: Three-dimensional contour maps for the total weight of the cables and inner struts, $W$, for the three loading combinations.

feasibility of the proposed method. The following conclusions were drawn:

(1) The values of vector height (i.e., $d_{1}$ ) and sag height (i.e., $d_{2}$ ) significantly affect the weight of the components and the prestress of a PUSC. Because the geometric stiffness of the structure increases with increasing rise-to-span and sag-tospan ratios, the areas of the cables decrease, and the weight of all the cables decreases correspondingly, whereas the slenderness ratio of the inner strut increases with increasing $d_{1}$ and $d_{2}$, leading to an increase in the sectional area and weight of the inner struts. In particular, when the appropriate combination of $d_{1}$ and $d_{2}$ is found, the material properties of the cables and the inner strut are optimized, thus reducing the total weight.

(2) The size of the external load has a major impact on the design of PUSCs. A greater total external load requires 


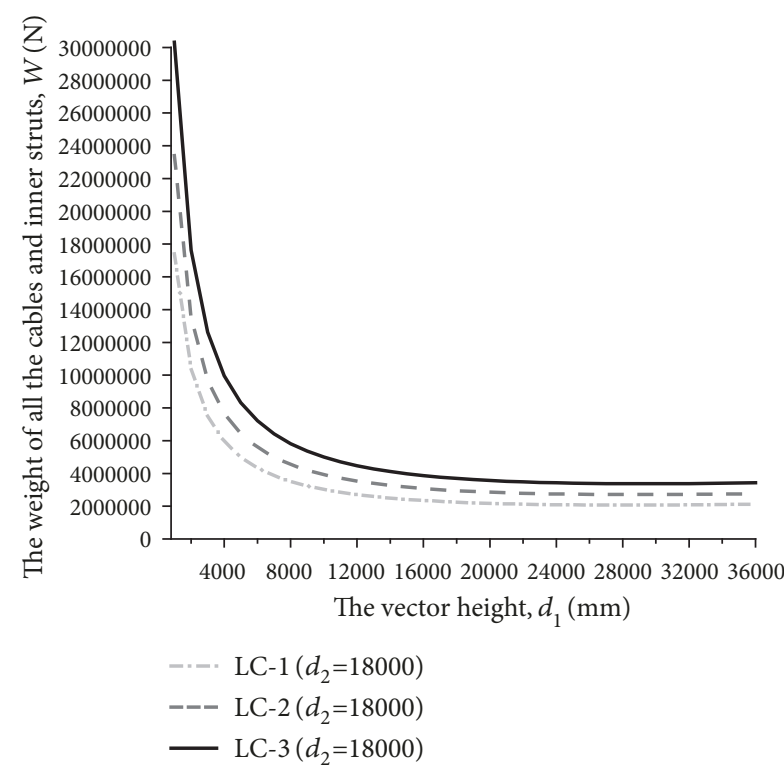

(a)

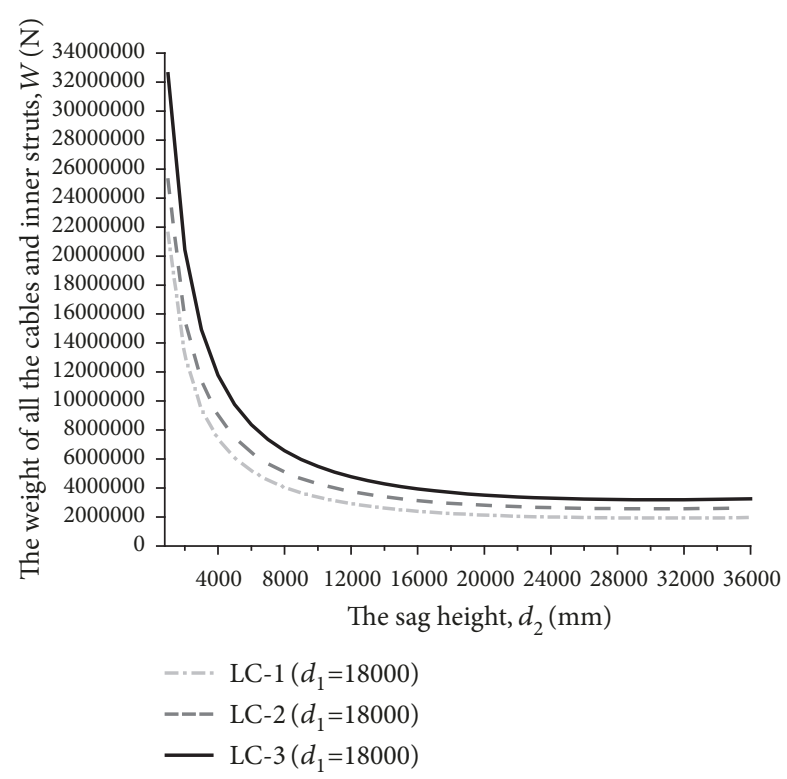

(b)

FIGURE 10: Two-dimensional contour maps for the weight of all the cables and inner struts, $W$, for the three loading combinations. (a) Variation in $W$ with various values of $d_{1}\left(1000 \leq d_{1} \leq 360000\right)$. (b) Variation in $W$ with various values of $d_{2}\left(1000 \leq d_{2} \leq 360000\right)$.

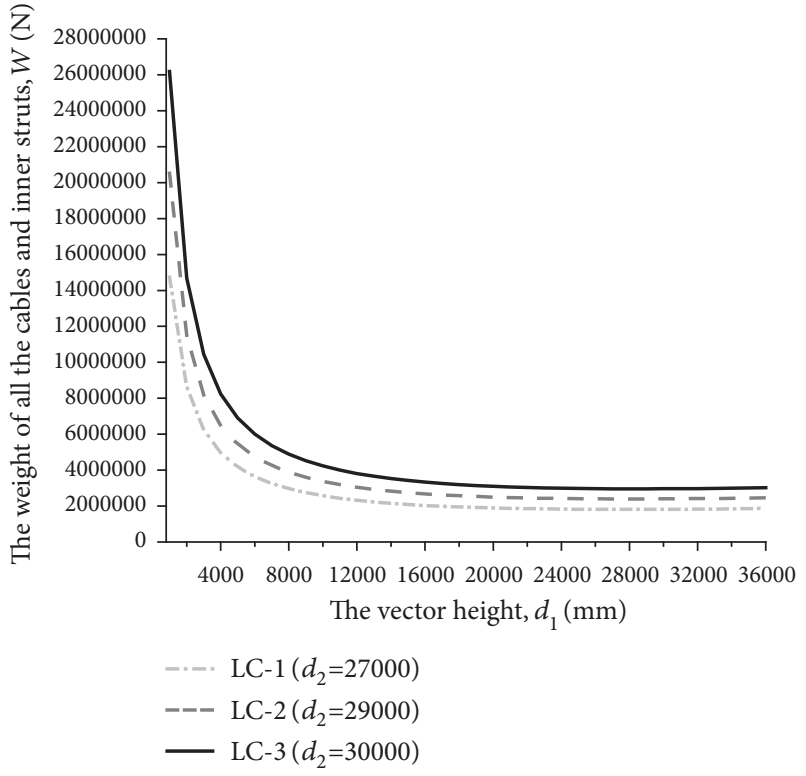

(a)

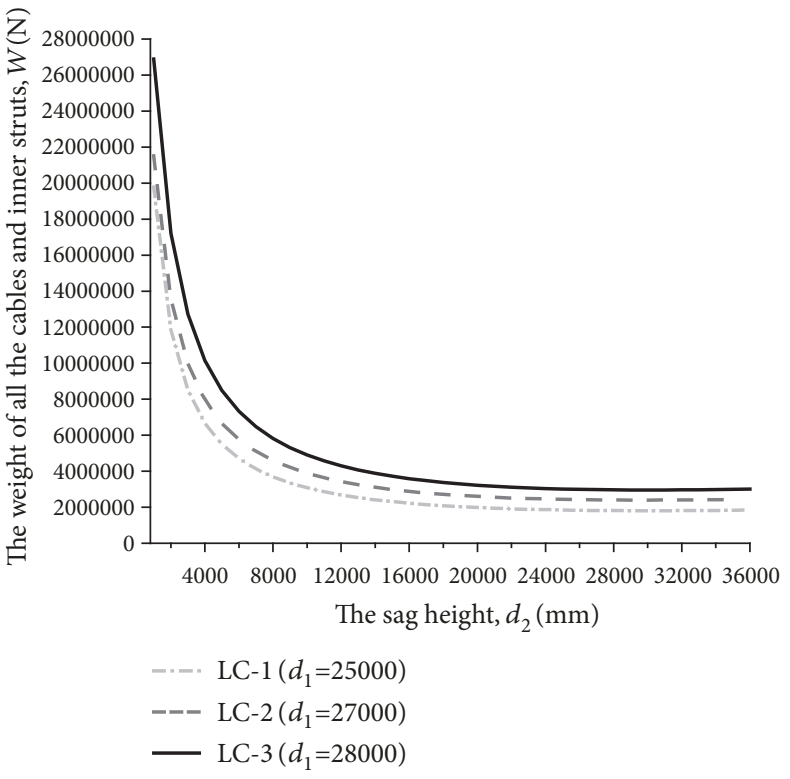

(b)

Figure 11: Two-dimensional contour maps for the weight of all the cables and struts, $W$, of the rational models. (a) Variation in $W$ with various values of $d_{1}\left(1000 \leq d_{1} \leq 360000\right)$. (b) Variation in $W$ with various values of $d_{2}\left(1000 \leq d_{2} \leq 360000\right)$.

greater $d_{1}$ and $d_{2}$ to increase the overall stiffness; therefore, the initial pretension forces of the cables, the sectional areas of the cables, the dimensions of the inner struts, and the total weight of all the cables and struts, $W$, are greater.

(3) The variation in $W$ exhibits three stages under external loads. For a consistent value of $d_{2}$ ( or $\left.d_{1}\right), W$ decreases sharply from a relatively high value during the initial increasing stage of $d_{1}$ (or $d_{2}$ ) and then starts to decrease gradually in the second stage. After reaching the lowest value, $W$ slowly increases during the last stage.

(4) By comparing the specifications of the components and the prestress of the entire structure, the optimal design 
mode, with an adequate geometric stiffness and a suitable prestress, was determined to fulfill the requirements of all the loading combinations.

\section{Nomenclature}

$A_{d}$ : Sectional area of diagonal cables

$A_{d, p w}$ : Sectional area of diagonal cables for the pressure wind loading condition

$A_{d, s w}$ : Sectional area of diagonal cables for the suction wind loading condition

$A_{d, v}$ : Sectional area of diagonal cables for the vertical loading condition

$A_{d, v p}$ : Sectional area of diagonal cables for the condition considering both pressure wind load and vertical loads

$A_{d, v s}$ : Sectional area of diagonal cables for the condition considering both suction wind load and vertical loads

$A_{d i}$ : Sectional area of diagonal cables of group- $i(i=1,2,3 \ldots)$

$A_{d i}(0)$ : Sectional area of diagonal cables of group- $i$ in the initial state $(i=1,2,3 \ldots)$

$A_{d i}(1)$ : Sectional area of diagonal cables of group $-i(i=1,2,3 \ldots)$ in the 1 st iteration

$A_{d i}(k)$ : Sectional area of diagonal cables of group $-i(i=1,2,3 \ldots)$ in the $k$ th $(k=$ $2,3,4 \ldots)$ iteration

$A_{s}$ : $\quad$ Sectional area of inner struts

$A_{s i}(0)$ : Sectional area of inner struts of group- $i$ ( $i$ $=1,2,3 \ldots)$ in the initial state

$A_{s i}(1)$ : Sectional area of inner struts of group- $i$ ( $i$ $=1,2,3 \ldots)$ in the 1st iteration

$A_{s i}(k)$ : Sectional area of inner struts of group- $i$ ( $i$ $=1,2,3 \ldots)$ in the $k$ th $(k=2,3,4 \ldots)$ iteration

$A_{r}$ : Sectional area of ridge cables

$A_{r, p w}$ : Sectional area of ridge cables for the pressure wind loading condition

$A_{r, s w}$ : Sectional area of ridge cables for the suction wind loading condition

$A_{r, v}$ : Sectional area of ridge cables for the vertical loading condition

$A_{r, v p}$ : Sectional area of ridge cables for the condition considering pressure wind load and vertical loads

$A_{r, v s}: \quad$ Sectional area of ridge cables for the condition considering suction wind load and vertical loads

$A_{r i}$ : Sectional area of ridge cables of group- $i$ ( $i$ $=1,2,3 \ldots$.)

$A_{r i}(0)$ : Sectional area of ridge cables of group- $i$ ( $i$ $=1,2,3 \ldots$. ) in the initial state

$A_{r i}(1)$ : Sectional area of ridge cables of group- $i$ ( $i$ $=1,2,3 .$. ) in the 1st iteration

$A_{r i}(k)$ : Sectional area of ridge cables of group- $i$ ( $i$ $=1,2,3 \ldots)$ in the $k$ th $(k=2,3,4 \ldots)$ iteration

$D^{\prime}$ : $\quad$ Self-weight of the PUSC

$d_{1}$ : $\quad$ Vector height

$d_{1, i}$ : Vector height of group- $i(i=1,2,3 \ldots)$ $d_{2}: \quad$ Sag height

$d_{2, i}: \quad$ Sag height of group- $i(i=1,2,3 \ldots)$

$E: \quad$ Elastic modulus of cables

$f$ : Maximum local deformation of the ridge cables

$f_{\text {con }}$ Limited local displacement of the ridge cables

$f_{p w}$ : Maximum local deformation of the ridge cables for the pressure wind loading condition

$f_{s w}$ : Maximum local deformation of the ridge cables for the suction wind loading condition

$f_{v}$ : Maximum local deformation of the ridge cables for the vertical loading condition

$f_{v p}$ : Maximum local deformation of the ridge cables for the condition considering both pressure wind load and vertical loads

$f_{v s}: \quad$ Maximum local deformations of the ridge cables for the condition considering both suction wind load and vertical loads

g: $\quad$ Gravitational acceleration, defined as 9.8 $\mathrm{N} / \mathrm{kg}$

$H_{d 1}$ : Horizontal component of $T_{d 1}$

$H_{r 1}$ : Horizontal component of $T_{r 1}$

$H_{r 1, p w}$ : Horizontal component of ridge cable force for the pressure wind loading condition

$H_{r 1, s w}$ : Horizontal component of ridge cable force for the suction wind loading condition

$H_{r 1, v}$ : Horizontal component of initial force of the ridge cables for the vertical loading condition

$H_{r 1, v p}$ : Horizontal component of initial force of the ridge cables for the condition considering both pressure wind load and vertical loads

$H_{r 1, v s}$ : Horizontal component of initial force of the ridge cables for the condition considering both suction wind load and vertical loads

$H_{r 2}$ : Horizontal component of $T_{r 2}$

$H_{r 2, p w}$ : Horizontal component of the force of ridge cables for the pressure wind loading condition

$H_{r 2, s w}$ : Horizontal component of the force of ridge cables for the suction wind loading condition

$H_{r 2, v}$ : Horizontal component of the force of ridge cables for the vertical loading condition

$H_{r 2, v p}$ : Horizontal component of the force of ridge cables for the condition considering both pressure wind load and vertical loads

$H_{r 2, v s}$ : Horizontal component of the force of ridge cables for the condition considering both suction wind load and vertical loads

L: $\quad$ Half of the horizontal span of the simplified calculation model

$L^{\prime}$ : $\quad$ Roofing live load of the PUSC

$N$ : Axial compressive load of the inner struts 
$n: \quad$ Number of substructures of the PUSC

$P: \quad$ Negative value of the virtual work performed by the load on the possible displacement

$P^{\prime}$ : $\quad$ Prestress forces of the PUSC

$q_{p w}: \quad$ Equivalent pressure wind line load

$q_{s w}: \quad$ Equivalent suction wind line load

$q_{v}: \quad$ Equivalent vertical line load

$q_{v}(0)$ : Initial uniform vertical line load, without considering the weight of the cables and struts

$q_{v}(1)$ : Uniform vertical line load applied to the ridge cables in the 1st iteration

$q_{v}(k)$ : Uniform vertical line load applied to the ridge cables in the $k$ th iteration $(k=$ 2,3,4...)

$R: \quad$ External load added on the PUSC

$T_{d 1}$ : Force of the diagonal cables in the initial prestress state

$T_{d 1, i}: \quad$ Force of the diagonal cables of group $-i(i=$ $1,2,3 \ldots)$ in the initial prestress state

$T_{d 2}$ : Forces of diagonal cables in the loading equilibrium state

$T_{d 2, p w}$ : Force of the diagonal cables for the pressure wind loading condition

$T_{d 2, s w}$ : Force of the diagonal cables for the suction wind loading condition

$T_{d 2, v}: \quad$ Forces of diagonal cables for the vertical loading condition

$T_{d 2, v p}$ : Forces of diagonal cables for the condition considering both pressure wind and vertical loads

$T_{d 2, v s}:$ Forces of diagonal cables for the condition considering both suction wind load and vertical loads

$T_{r 1}$ : Force of the ridge cables in the initial prestress state

$T_{r 1, i}: \quad$ Force of the ridge cables of group- $i(i=$ $1,2,3 \ldots)$ in the initial prestress state

$T_{r 2}$ : Force of ridge cables in the loading equilibrium state

$T_{r 2, p w}$ : Force of the ridge cables for the pressure wind loading condition

$T_{r 2, s w}$ : Force of the ridge cables for the suction wind loading condition

$T_{r 2, v}: \quad$ Forces of ridge cables for the vertical loading condition

$T_{r 2, v p}:$ Forces of ridge cables for the condition considering both pressure wind load and vertical loads

$T_{r 2, v s}:$ Forces of ridge cables for the condition considering both suction wind load and vertical loads

$T_{s 1}: \quad$ Compression force on the inner struts in the initial prestress state

$T_{s 2}$ : Compression force of the inner struts in the loading condition
$T_{s 2, i}: \quad$ Compression force of the inner struts in the loading condition of group $-i(i=$ $1,2,3 \ldots)$

$T_{s 2, i}(1)$ : Compression force of the inner struts in the loading condition of group $-i(i=$ $1,2,3 \ldots)$ in the 1st iteration

$T_{s 2, i}(k)$ : Compression force of the inner struts in the loading condition of group $-i(i=$ $1,2,3 \ldots)$ in the $k$ th $(k=2,3,4 \ldots)$ iteration

$U: \quad$ Total strain energy of all the cable elements under a possible displacement

$u_{d, v}: \quad$ Deformation of the diagonal cables in the vertical loading condition

$u_{r, v}$ : Deformation of the ridge cables in the vertical loading condition

$u_{r 1, v}: \quad$ Deformation of the ridge cables, caused by $w_{v}$

$u_{r 2, v}: \quad$ Deformation of the ridge cables, caused by

$W: \quad f_{v}$

$W_{\text {cable: }}$ Weight of all the cables

$W_{\text {rational }}$ : Weight of all the cables and struts for the rational mode

$W_{\text {strut }}: \quad$ Weight of all the inner struts

$W_{i}: \quad$ Weight of the cables and struts of group $-i$ $(i=1,2,3 \ldots)$

$W_{i}(0)$ : Weight of the cables and struts of group- $i$ $(i=1,2,3 \ldots)$ in the initial state

$W_{i}(1)$ : Weight of the cables and struts of group- $i$ $(i=1,2,3 \ldots)$ in the 1st iteration

$W_{i}(k)$ : Weight of the cables and struts of group $-i$ $(i=1,2,3 \ldots)$ in the $k$ th $(k=2,3,4 \ldots)$ iteration

$W^{\prime}$ : Wind load of the PUSC

$w$ : $\quad$ Vertical deformations of the inner strut

$w_{0}$ : Basic wind pressure

$w_{\text {con }}: \quad$ Limited mid-span displacement of the entire structure

$w_{k}: \quad$ Value of wind load

$w_{p w}$ : Vertical deformations of the inner strut for the pressure wind loading condition

$w_{s w}$ : Vertical deformations of the inner strut for the suction wind loading condition

$w_{v}$ : Vertical deformations of the inner strut for the vertical loading condition

$w_{v p}$ : Vertical displacement of the inner strut for the condition considering both pressure wind load and vertical loads

$w_{v s}: \quad$ Vertical displacement of the inner strut for the condition considering both suction wind load and vertical loads

$x: \quad$ Value of the $x$-coordinate

$Z_{r}: \quad z$-coordinate of the ridge cables

$Z_{d}: \quad z$-coordinate of the diagonal cables

П: $\quad$ Total potential energy of all the cable elements for PUSC

$\Delta l_{d}: \quad$ Total elongation of a diagonal cable after loading 
$\Delta l_{d, v}$ : Elongation of a diagonal cable for the vertical loading condition

$\Delta l_{r}: \quad$ Total elongation of a ridge cable after loading

$\Delta l_{r, p w}$ : Elongation of a ridge cable in the pressure wind loading condition

$\Delta l_{r 1, p w}$ : Elongation of a ridge cable, caused by $w_{s w}$

$\Delta l_{r 2, p w}$ : Elongation of a ridge cable, caused by $f_{s w}$

$\Delta l_{r, s w}$ : Elongation of a ridge cable in the suction wind loading condition

$\Delta l_{r 1, s w}$ : Elongation of a ridge cable, caused by $w_{s w}$

$\Delta l_{r 2, s w}$ : Elongation of a ridge cable, caused by $f_{s w}$

$\Delta l_{r, v}$ : Elongation of a ridge cable in the vertical loading condition

$\Delta l_{r 1, v}$ : Elongation of a ridge cable, caused by $w_{v}$

$\Delta l_{r 2, v}$ : Elongation of a ridge cable, caused by $f_{v}$

$\Delta T_{r}$ : Increments of the forces on ridge cables

$\Delta T_{d}: \quad$ Increments of the forces on diagonal cables

$\Delta W_{i}(1)$ : Absolute value of the variation of $W_{i}(1)(i$ $=1,2,3 \ldots)$

$\Delta W_{i}(k)$ : Absolute value of the variation of $W_{i}(k)(i$ $=1,2,3 \ldots ; k=2,3,4 \ldots)$

$\Delta W_{\text {lim }}:$ The permissible variation of $W$

$\Delta X: \quad$ Increment of real solution of the structural displacement

$\sigma_{\text {con }}: \quad$ Design strength of cables

$\sigma_{y}: \quad$ Yield strength of the inner struts

$\varphi: \quad$ Stability factor of the inner struts

$\rho_{c}: \quad$ Density of the cables

$\rho_{s}$ : Density of the inner struts

$\mu_{s}: \quad$ Shape coefficient

$\mu_{z}: \quad$ Wind-pressure height coefficient

$\beta_{z}$ : Wind vibration coefficient.

\section{Data Availability}

The data used to support the findings of this study are included within the article.

\section{Conflicts of Interest}

The authors declare that there are no conflicts of interest regarding the publication of this paper.

\section{Acknowledgments}

This work is supported by the National Natural Science Foundation of China (Grant no. 11673039) and The Natural Science Foundation of the Jiangsu Higher Education Institutions of China (Grant no. 18KJB560011).

\section{References}

[1] B. Luo, Z. Guo, X. Chen, F. Gao, and K. Wang, "Static equilibrium form-finding analysis of cable-strut system based on nonlinear dynamic finite element method," Advanced Steel Construction, vol. 11, no. 4, pp. 452-468, 2015.
[2] B. Duan, Y. Qiu, F. Zhang, and B. Zi, "Analysis and experiment of the feed cable-suspended structure for super antenna," in Proceedings of the IEEE/ASME International Conference on Advanced Intelligent Mechatronics, pp. 329-344, IEEE, Washington, USA, 2008.

[3] Y. Xu, F. Guan, X. Xu, H. Wang, and Y. Zheng, "Development of a novel double-ring deployable mesh antenna," International Journal of Antennas \& Propagation, vol. 2012, Article ID 375463, 10 pages, 2012.

[4] P. Allingham, "Urban space, representation, and artifice," Knowledge Technology \& Policy, vol. 21, no. 4, pp. 163-174, 2008.

[5] A. T. Daloglu, M. Artar, K. Özgan, and A. İ. Karakas, "Optimum design of steel space frames including soil-structure interaction," Structural and Multidisciplinary Optimization, vol. 54, no. 1, pp. 117-131, 2016.

[6] B. Y. Duan, Y. Y. Qiu, F. S. Zhang, and B. Zi, "On design and experiment of the feed cable-suspended structure for super antenna," Mechatronics, vol. 19, no. 4, pp. 503-509, 2009.

[7] S. Ohkubo, Y. Watada, and F. Toshio, "Nonlinear analysis of truss by energy minimization," Computers \& Structures, vol. 27, no. 1, pp. 129-145, 1987.

[8] Y. C. Toklu, G. Bekdaş, and R. Temur, "Analysis of trusses by total potential optimization method coupled with harmony search," Structural Engineering and Mechanics, vol. 45, no. 2, pp. 183-199, 2013.

[9] R. Temür, Y. S. Türkan, and Y. C. Toklu, "Geometrically nonlinear analysis of trusses using particle swarm optimization," in Recent Advances in Swarm Intelligence and Evolutionary Computation, vol. 585 of Studies in Computational Intelligence, pp. 283-300, Springer International Publishing, Cham, Switzerland, 2015.

[10] A. E. Charalampakis and I. Chatzigiannelis, "Analytical solutions for the minimum weight design of trusses by cylindrical algebraic decomposition," Archive of Applied Mechanics, vol. 88, no. 1-2, pp. 1-11, 2018.

[11] S. Pezeshk, "Design of framed structures: An integrated nonlinear analysis and optimal minimum weight design," International Journal for Numerical Methods in Engineering, vol. 41, no. 3, pp. 459-471, 1998.

[12] H. Kawamura, H. Ohmori, and N. Kito, "Truss topology optimization by a modified genetic algorithm," Structural and Multidisciplinary Optimization, vol. 23, no. 6, pp. 467-473, 2002.

[13] H.-J. Schek, "The force density method for form finding and computation of general networks," Computer Methods Applied Mechanics and Engineering, vol. 3, no. 1, pp. 115-134, 1974.

[14] R. Motro, S. Najari, and P. Jouanna, "Static and dynamic analysis of tensegrity systems," in Proceedings of the International Symposium on Shell and Spatial Structures: Computational Aspects, vol. 26 of Lecture Notes in Engineering, pp. 270-279, Springer International Publishing, Berlin, Germany, 1987.

[15] Y. Luo and S. Dong, "Nonlinear force method analysis for space truss with mobile mechanisms," Acta Mechanica Solida Sinica, vol. 23, no. 3, pp. 288-294, 2002.

[16] J. Zhou, W. Chen, B. Zhao, and S. Dong, "A feasible symmetric state of initial force design for cable-strut structures," Archive of Applied Mechanics, vol. 87, no. 8, pp. 1385-1397, 2017.

[17] H. Deng, T. Li, Z. Wang, and X. Ma, "Pretension design of space mesh reflector antennas based on projection principle," Journal of Aerospace Engineering, vol. 28, no. 6, p. 04014142, 2015.

[18] F. Liu, H. J. Li, and T. C. Wang, "Energy principle and nonlinear electric-mechanical behavior of ferroelectric ceramics," Acta Mechanica, vol. 198, no. 3-4, pp. 147-170, 2008. 
[19] A. Juozapaitis and A. Norkus, "Displacement analysis of asymmetrically loaded cable," Journal of Civil Engineering and Management, vol. 10, no. 4, pp. 277-284, 2004.

[20] Code for Design of Steel Structure, China Architecture \& Building Press, GB 50017-2017.

[21] Load Code for the Design of Building Structures, China Architecture \& Building Press, GB50009-2012. 


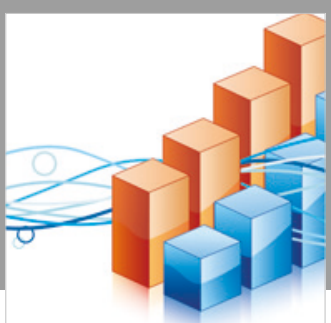

Advances in

Operations Research

\section{-n-m}
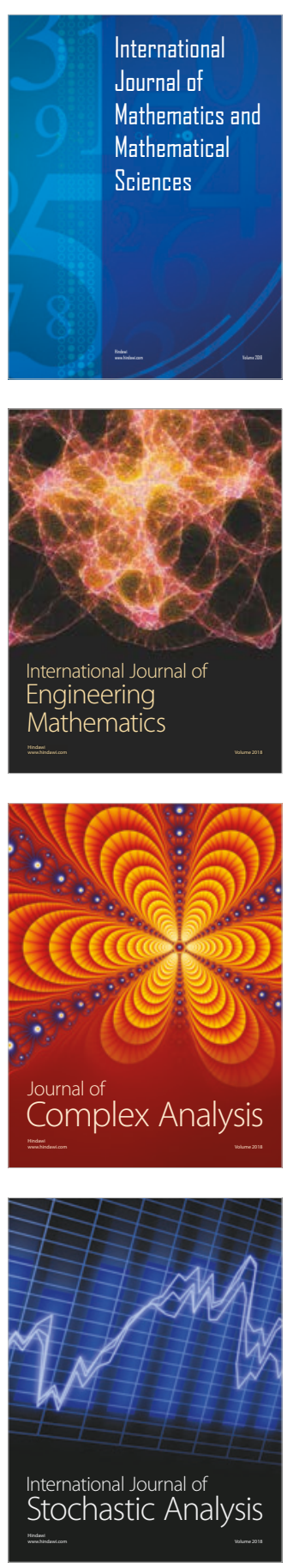
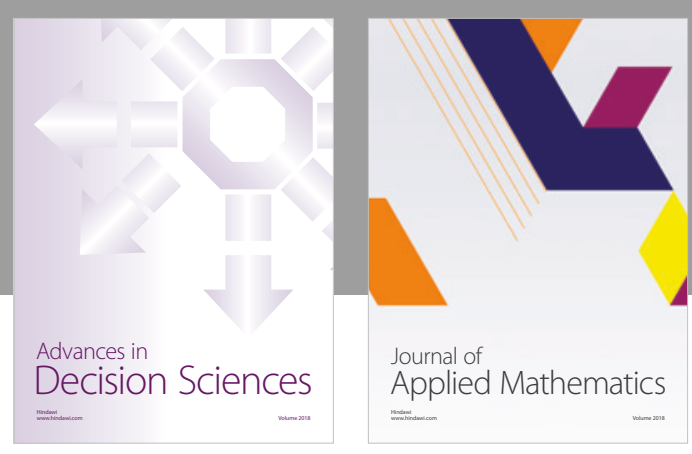

Journal of

Applied Mathematics
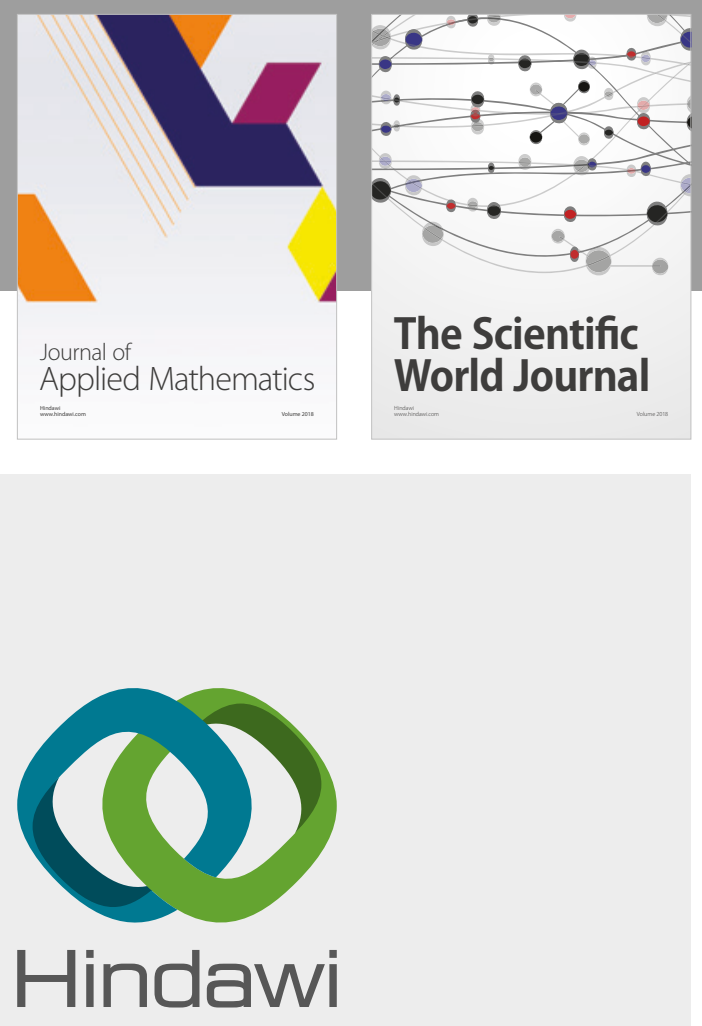

Submit your manuscripts at

www.hindawi.com

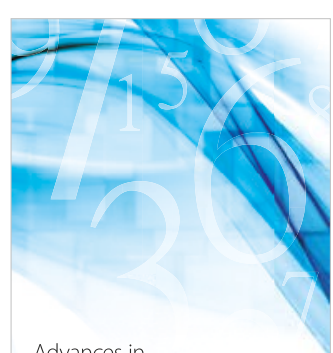

Advances in
Numerical Analysis
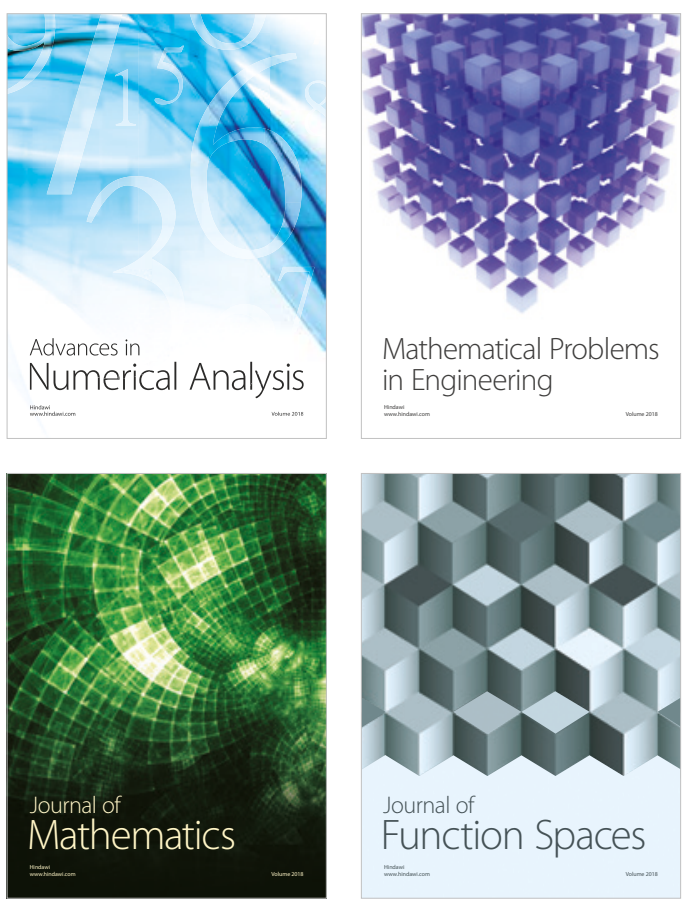

Mathematical Problems in Engineering

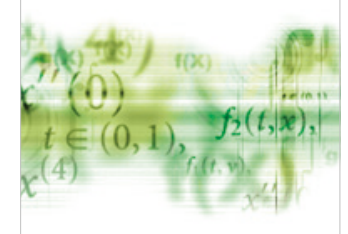

International Journal of

Differential Equations

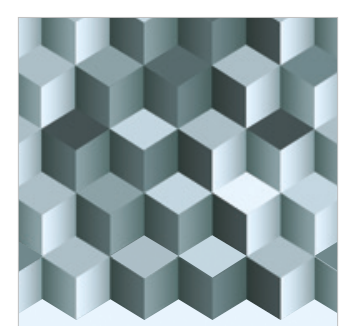

Journal of

Function Spaces

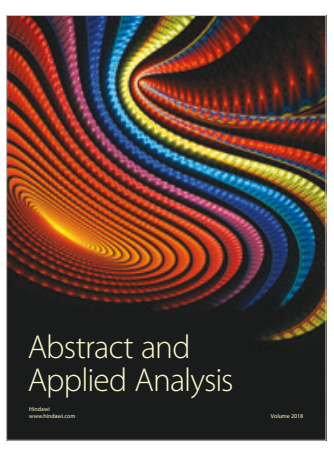

The Scientific

World Journal

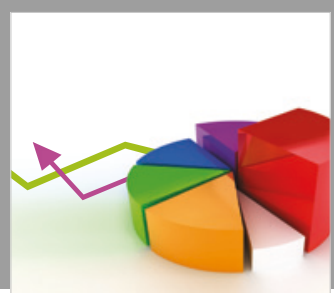

Journal of

Probability and Statistics
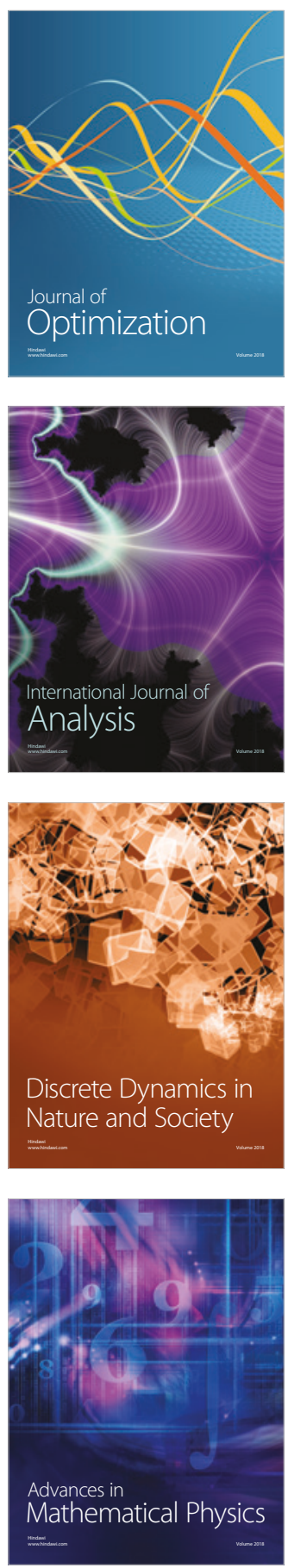